Revue

Revue de l'histoire des religions

del'histoire des religions

$3 \mid 2019$

Varia

\title{
Trismégistos. Circulation et contextes d'un nom divin
}

Trismegistos: the circulation and contexts of a divine name

\section{Florian Audureau}

\section{CpenEdition \\ Journals}

Édition électronique

URL : https://journals.openedition.org/rhr/9884

DOI : $10.4000 /$ rhr.9884

ISSN : 2105-2573

\section{Éditeur}

Armand Colin

\section{Édition imprimée}

Date de publication : 1 septembre 2019

Pagination : 475-506

ISBN : 978-2-200-93260-2

ISSN : 0035-1423

Référence électronique

Florian Audureau, «Trismégistos. Circulation et contextes d'un nom divin », Revue de l'histoire des religions [En ligne], 3 | 2019, mis en ligne le 01 janvier 2022, consulté le 08 janvier 2022. URL : http:// journals.openedition.org/rhr/9884; DOI : https://doi.org/10.4000/rhr.9884 


\section{Trismégistos Circulation et contextes d'un nom divin}

À la fin de l'antiquité, le nom divin d'Hermès trismégistos circule dans plusieurs langues et dans des contextes énonciatifs différents. On en cite généralement les occurrences dans le cadre d'une histoire de l'hermétisme pour établir une continuité du dieu à travers ses actualisations. L'article se propose donc d'effectuer une revue de ces documents pour analyser le nom à partir de ses contextes énonciatifs. En effet, l'usage du nom nous informe sur les dynamiques sociales en jeu dans son énonciation, car il ne recouvre pas toujours les mêmes réalités. On mettra ainsi en lumière d'un côté un usage rituel, avec l'évocation d'une divinité locale parmi d'autres, de l'autre un usage identitaire, où le nom donne du pouvoir à des identités sociales.

\section{Trismegistos : the circulation and contexts of a divine name}

In late Antiquity, the divine name of Hermes Trismegistos circulates in various languages and different enunciative contexts. It is generally quoted with respect to the history of Hermeticism as a way of postulating continuity between the diverse instances of this god. This article aims at analyzing these occurrences of the divine name in light of their various enunciative contexts. It provides insight into the social dynamics that are at play in its utterance, for its use does not always imply the same social phenomena. This analysis will thus shed light both on its ritualistic use, when it invokes a local divinity, as well as its identity use, when it serves to empower new religious and scientific identities. 
Qui dit Trismégiste dit aussitôt Hermès. Cette épithète, qui fait figure de «nom propre $»^{1}$ pour Festugière, est parfois prise pour une signature infaillible de l'hermétisme, ou du moins pour un signe fort. Elle prend alors valeur d'épiclèse, si bien que dès qu'il s'agit d'interroger les origines de l'hermétisme, les commentateurs en citent les occurrences dans une série de documents. Le nom divin permettrait ainsi de repérer une unité religieuse. D'un point de vue purement linguistique, l'origine et la traduction de cet adjectif en grec ont eux-mêmes fait l'objet d'un débat parmi les égyptologues dans les années 1970 et Jan Quaegebeur a donné le dossier le plus complet des pièces grecques, démotiques et hiéroglyphiques tout en retraçant l'historique des interprétations

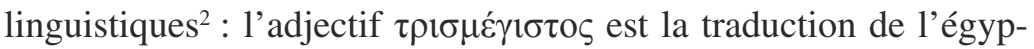
tien $\varepsilon_{3} \varepsilon_{3} \varepsilon_{3} w r\left(s_{3}\right.$ signifiant « grand» et $w r$ servant d'adverbe de superlatif) ${ }^{3}$. Littéralement, l'expression $\varsigma_{3} \varsigma_{3} w r$, que l'on donnait autrefois pour le prototype égyptien, serait donc plutôt un «deux fois très grand $»^{4}$. On connaît aussi des formules $\varsigma_{3} \varsigma_{3} \varsigma_{3}$ qui correspondent à la traduction grecque par $\tau$ í́ $\sigma \mu \varepsilon \gamma \alpha \varsigma^{5}$. Enfin, le dossier égyptien comprend également une répétition de cinq fois le terme $\varsigma_{3}$ que Ritner rendait par «pentamegistos $»^{6}$. Le Trismégiste apparaît donc dans un spectre assez large d'usages (simple gémination de l'adjectif selon un formulaire invocatoire égyptien, répétitions multiples, ajout d'un adverbe intensificateur).

1. André-Jean Festugière, La révélation d'Hermès Trismégiste I, Paris, LecoffreGabalda, 1944, p. 74.

2. Jacques Parlebas, «L'origine égyptienne de l'appellation Hermès Trismegistos », Göttinger Miszellen [GM] 13, 1974, p. 25-28; Philippe Derchain et Maria-Theresia Derchain, « Noch einmal "Hermes Trismegistos" », GM 15, 1975, p. 7-10. Robert K. Ritner, «Hermes Pentamegistos », GM 49, 1981, p. 73-75 ; Garth Fowden, The Egyptian Hermes : a historical approach to the late pagan mind, Cambridge, 1986, p. 216-217 ; Jan Quaegebeur, « Thot-Hermès, le dieu le plus grand ! », Hommages à François Daumas, Montpellier, Institut d'Égyptologie, Université Paul Valéry, 1986, p. 525-544.

3. Quaegebeur, art. cit. Le terme $w r$ peut à la fois servir d'adjectif signifiant « grand » et d'adverbe d'intensification («très », «beaucoup »). Notons que la forme égyptienne la plus courante est $\varsigma_{3} \varsigma_{3}$, voir Lexikon der ägyptischen Götter und Götterbezeichnnungen II, 16.

4. ibid. p. 528-529.

5. loc. cit.

6. Ritner, art. cit. 
Pour Quaegebeur, le trois fois (très) grand ne se distingue donc pas vraiment du deux fois (très) grand ${ }^{7}$.

À la suite de cet examen linguistique qui a relevé la fluidité des traductions, le présent article va envisager les différentes occurrences disponibles dans leur contexte énonciatif. En partant des variantes formelles de l'adjectif, cette revue cherchera à analyser les dynamiques d'usage du nom divin dans divers milieux sociaux. Ce faisant, l'objectif est de contribuer à une meilleure compréhension des modalités d'apparition de l'hermétisme en tant que courant philosophico-religieux.

Le développement de l'article suivra une progression en trois temps. Tout d'abord, je reviendrai sur les problèmes de définitions de l'hermétisme et sur la question de sa frontière avec la magie depuis une perspective théorique. Ensuite, l'examen des occurrences du terme $\tau \rho \iota \sigma \mu \varepsilon ́ \gamma \imath \sigma \tau o \varsigma / \tau \rho i ́ \sigma \mu \varepsilon \gamma \alpha \varsigma$ permettra d'envisager des Hermès trois fois (très) grand sans hermétisme à l'époque romaine. À rebours, la dernière section s'appuiera sur des occurrences manifestement hermétiques pour les resituer dans les dynamiques sociales et intellectuelles contemporaines.

\section{Frontières D'Un CONCEPT}

En fonction des auteurs, l'hermétisme peut osciller entre une définition minimale et une définition maximale, ce qui confère à la catégorie un certain flou ${ }^{8}$. Au maximum, l'hermétisme est employé par les modernes comme catégorie analytique pour englober un ensemble plus ou moins unifié de savoirs et de pratiques (comme l'astrologie, la magie et l'alchimie) et leur transmission au cours des siècles. Au minimum, l'hermétisme constitue une catégorie historique particulière pour désigner a) un ensemble documentaire multilingue (grec, latin, arménien et copte) ${ }^{9}$ et la

7. Quaegebeur, art. cit. p. 537.

8. Kevin Thomas van Bladel, The Arabic Hermes: from pagan sage to prophet of science, Oxford University Press, 2009, p. 17-22 ; « Calling something Hermetic $[\ldots]$ does more to obscure than to explain its meaning and history », ibid. p. 20.

9. Pour une présentation exhaustive des textes, voir Anna Van den Kerchove, La voie d'Hermès. Pratiques rituelles et traités hermétiques, Leiden, Brill, 2012, p. 1-13. 
doctrine philosophique attenante, où la révélation d'Hermès-Thot et sa transmission par écrit font figure de pierre angulaire, et b) les ramifications de cette doctrine sous l'autorité pseudépigraphique d'Hermès Trismégiste dans la littérature médiévale. Un problème de la catégorie analytique, c'est sa diversité, qui englobe en un même ensemble "ésotérique » des savoirs qui ont surtout en commun d'être parfois rejetés par l'institution religieuse dominante. Le concept permet alors de subsumer une diversité de savoirs sous une même catégorie sans nécessairement interroger leurs rapports mutuels. La catégorie historique se concentre quant à elle sur l'idée de l'identification et de la transmission philologique pour envisager les processus de transferts culturels et historiques, quitte à ajouter des articulations (Egyptian Hermes, Arabic Hermes, Hermes Latinus...). Un problème posé par cette seconde catégorie, c'est qu'elle se focalise souvent sur la dimension intellectuelle et doctrinale, abstraction faite des rituels. Or, l'hermétisme antique n'est pas qu'une théorie philosophique sur le monde, ce sont aussi des rites et des pratiques religieuses ${ }^{10}$. À mi-chemin entre ces cas extrêmes, on pourra identifier plusieurs déclinaisons de l'hermétisme permettant d'inclure dans le corpus certains rituels ou certains documents qui se démarquent de l'ensemble plus large.

Dans le même ordre d'idées, la question des rapports entre l'hermétisme et les rituels, en particulier la magie, reconduit un débat en cours depuis plusieurs décennies, sur la distinction entre religion et magie d'un point de vue conceptuel ${ }^{11}$. Le cas de l'Égypte y est particulièrement sensible puisque la religion institutionnelle est elle-même de nature «magique » et qu'aucune

10. ibid., p. 13-18.

11. La bibliographie sur ce sujet est si longue que je ne retiendrai ici que quelques travaux dans le champ des sciences de l'antiquité : Jonathan Z. Smith, Map is not territory: studies in the history of religions, Leiden, Brill, 1978 ; Fritz Graf, «Prayer in Magical and Religious Ritual », in Christopher A. Farone, Dirk Obbink (dirs.), Magika Hiera. Ancient Greek Magic and Religion, Oxford University Press, 1991, p. 188-213 ; Hendrik Simon Versnel, « Beyond Cursing : The Appeal to Justice in Judicial Prayers », in Magika Hiera, p. 60-106 et "Some reflexions on the relationship magic/religion», Numen 38/2, 1991, p. 177-197; Fritz Graf, "Excluding the Charming », in Marvin W. Meyer, Paul Allan Mirecki (dirs.), Ancient magic and ritual power, Boston, États-Unis d'Amérique, Brill, 2001, p. 29-42 ; C. A. Hoffmann, « Fiat Magia », in Marvin W. Meyer, Paul Allan Mirecki (dirs.), Magic and ritual in the ancient world, Brill, 2002, p. 179-194 ; Bernd-Christian Otto, «Towards Historicizing "Magic" in Antiquity », Numen 60, 2013, p. 308-347. 
distinction sociologique ne peut être établie pour démarquer les pratiques «religieuses» des pratiques « magiques $»^{12}$. C'est en s'appuyant sur cet héritage culturel et conceptuel que prend forme l'hermétisme antique en tant que catégorie moderne.

Dans le discours critique, il arrive parfois que magie et hermétisme se confondent lorsque l'historien passe insensiblement de la définition historique à la catégorie analytique. Par exemple, Fowden adopte une méthode historique pour repérer l'hermétisme (définition minimale), mais sa distinction entre hermétisme philosophique (le corpus hermeticum) et hermétisme technique (les magica des Papyri Graecae Magicae $[=P G M])^{13}$ reconduit la catégorie analytique de manière rétroactive: le devenir de l'hermétisme (sens minimum) aux époques postérieures (sens maximum) permet a posteriori de catégoriser en amont les pratiques analogues. Comme chez Festugière, la notion de "magie hermétique » devient alors très large. On est en droit de se demander si l'hermétisme savant n'a pas plutôt fourni tardivement un refuge et un espace de légitimation et de théorisation à de multiples pratiques devenues de mauvais aloi dans le paradigme des monothéismes. La magie est-elle hermétique ou le devientelle sous certaines conditions? Certes, il ne faut pas penser les deux phénomènes, les deux genres de textes comme radicalement distincts ou comme des mouvements parallèles dans la société ; mais autant il convient d'établir les affinités entre les deux corpus, autant il faut éviter d'utiliser des éléments singuliers pour englober la totalité d'un phénomène sous l'appellation de l'autre. Prenons la fameuse prière du papyrus Mimaut (PGM III.591-601), dont

12. Joachim Friedrich Quack, «La magie au temple », in Yvan Koenig (dir.), La magie en Égypte: à la recherche d'une définition. Actes du colloque les 29 et 30 septembre 2000, Paris, Documentation Française, 2002, p. 41-68; Núria Torras, «Funciones y habilidades del sacerdote puro de Sekhmet: rituales mágicos en el contexto del templo », in Emilio Suárez, Miriam Blanco, Eleni Chronopoulou \& Isabel Canzobre (dirs.), Magikè téchne. Formación y consideración social del mago en el Mundo Antiguo, Madrid, Dykinson, 2017, p. 15-32 ; Robert K. Ritner, "The Religious, Social, and Legal Parameters of Traditional Egyptian Magic », in Marvin W. Meyer, Paul Allan Mirecki (dirs.), Ancient magic and ritual power, Boston, Brill, 2001, p. 43-60.

13. Fowden, op. cit. p. 57-74, notamment p. 66-68; qu'il y ait continuité entre magie pharaonique et magie gréco-égyptienne avec une variation de style (p. 66), c'est une chose bien établie ; mais l'idée que la magie gréco-égyptienne constituait de facto l'hermétisme technique et était perçue comme foncièrement hermétique (p. 68) mérite d'être davantage étayée. 
on trouve une version latine dans l'Asclepius et une version copte dans le traité de Nag Hammadi VI. $7^{14}$ : la présence d'un même texte ne doit pas oblitérer la pragmatique, laquelle donne au texte des actualisations qui peuvent varier d'une situation à l'autre et introduit ipso facto des effets de variations. Trait d'union entre les deux corpus, la prière prend un sens différent du fait de son environnement contextuel : dans le corpus magique, elle apparaît dans une salutation au soleil pour l'obtention de multiples bienfaits, comme ce pouvait être le cas pour d'autres prières traditionnelles ; dans ses actualisations hermétiques, la connaissance devient le seul vœu exprimé envers le dieu ${ }^{15}$. Ici, la question des liens ne doit donc pas écarter celle des déformations. Aussi ne me semble-t-il pas possible de parler d'une "prière hermétique primitive » ${ }^{16}$. Pour ainsi dire, à moins de prouver que l'auteur du papyrus Mimaut a emprunté son texte directement à Hermès Trismégiste, la même prière est hermétique quand elle est actualisée dans le contexte de l'hermétisme ( $N H$ VI.7) et ne l'est pas quand elle se situe dans une pragmatique plus traditionnelle (papyrus Mimaut).

Deuxièmement, la confusion peut résulter de la présence du nom d'Hermès dans un document. Quand Arnouphis provoque la pluie en invoquant Hermès-Aérios lors des guerres de MarcAurèle, a-t-on besoin des catégories analytique ou historique pour rendre compte de l'évènement ${ }^{17}$ ? L'épigramme V.24 de Martial est-elle une parodie d'un hymne hermétique parce qu'y

14. Jean-Pierre Mahé, Hermès en Haute-Égypte I. Les textes hermétiques de Nag Hammadi et leurs parallèles grecs et latins, Québec, Presses de l'Université Laval, 1978, p. 137-167.

15. William C. Grese, «Magic in Hellenistic Hermeticism », in Hermeticism $\&$ the Renaissance : Intellectual History and the Occult in Early Modern Europe, éds. Ingrid Merkel et Allen G. Debus, Washington DC, The Folger Shakespeare Library, 1988, p. 51-55.

16. Mahé, Hermès en Haute-Égypte I, op. cit. p. 146. Je souligne.

17. Dion Cassius, Histoire Romaine LXXI.8.4 ; pour l'interprétation hermétique, voir Th. Mommsen, « Das Regenwunder der Marcus-Säule », Hermes 30 / 1, 1895, p. 103 ; Marta Sordi, « Le monete di Marco Aurelio con Mercurio e la pioggia miracolosa », Annali dell'Istituto Italiano di Numismatica V-VI, 1959-1958, p. 46 ; Pierre Cordier, « Dion Cassius et les phénomènes religieux "égyptiens" », in Laurent Bricault et Miguel John Versluys (éds.), Nile into Tiber. Egypt in the Roman World, 2007, p. 107-108; John Scheid, «Sujets religieux et gestes rituels sur la colonne Aurélienne. Questions sur la religion à l'époque de Marc Aurèle », in John Scheid, Valérie Huet (éds.), Autour de la colonne Aurélienne. Geste et image sur la colonne de Marc Aurèle à Rome, 2000, p. 235-236. Contra Julien Guey, «Encore la "pluie miraculeuse », Revue de philologie, de littérature et 
figure la qualification ter unus appliquée à un gladiateur homonyme du dieu ${ }^{18}$ ? La présence du nom d'Hermès sur un document magique syriaque suffit-elle pour y voir une influence de l'hermétisme $^{19}$ ? Le nom d'Hermès dans les $P G M$ doit-il être rapporté prioritairement au Trismégiste parce qu'il apparaît en contexte magique $^{20}$ ? Pour la catégorie restreinte, la présence du nom et celle du thème de la révélation sont prises comme conditions suffisantes. Aussi le PGM XXIVa devient-il un texte hermétique pour certains chercheurs, parce qu'il mentionne « une copie d'un livre sacré découvert dans les archives d'Hermès $»^{21}$, de même que le P. Berol. 21243 qui parle d'incantations trouvées «à Héliopolis, dans le livre sacré que l'on appelle [Livre] d'Hermès, dans le temple, en lettres égyptiennes et traduites en grec $»^{22}$. Pour ces deux textes, il s'agit de magie égyptienne au sens large. Et si on considère la fonction scribale de Thot comme caractéristique

d'histoire anciennes 22, 1948, p. 47-62 ; Garth Fowden, «Pagan versions of the rain miracle of A.D. $172 »$, Historia 36 / 1, 1987, p. 83-95.

18. H. S. Versnel, «A Parody on Hymns in Martial V 24 and Some Trinitarian Problems », Mnemosyne 27 / 4, 1974, p. 365-405 a montré qu'il s'agissait d'une invention comique parfaitement compréhensible à partir de références linguistiques classiques.

19. Philippe Gignoux, Incantations magiques syriaques, Louvain, Peeters, 1987, p. 25-43 pour le texte et la traduction; Kevin Thomas van Bladel, op. cit., p. 25-26 le cite dans le cadre de l'hermétisme ; il s'avère que le nom apparaît bien une fois sous la forme hrmws (1. 26) et deux fois dans des reconstitutions hypothétiques mais la référence est à la mythologie grecque et au dieu d'Argos (hrgws).

20. Par exemple, Hans Georg Gundel, Weltbild und Astrologie in den griechischen Zauberpapyri, München, Beck, 1968, p. 26 fait du dieu lunaire Hermès une actualisation du Trismégiste; de même Fowden, The Egyptian Hermes, op. cit., p. 25, n. 68 cite Papyri Graecae Magicae [PGM], V.400-421

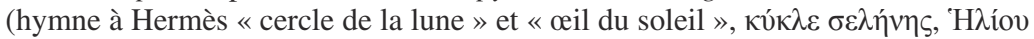
ò $\varphi \theta \alpha \lambda \mu \varepsilon ́), c f . P G M$ VII.668-685 et XVIIb. Plus récemment, Florian Ebeling, The secret history of Hermes Trismegistus : hermeticism from ancient to modern times, London, Cornell University Press, 2007, p. 24-25 (PGM V.370-445, hymne d'invocation à Thot-Hermès donné comme exemple de l'hermétisme dans la magie) et Monaca Mariangelaca, "Ermete e la divinazione nei Papyri Graecae Magicae », Hermetism. From Late Antiquity to Humanism. La tradizione ermetica dal mondo tardo-antico all'umanesimo. Atti del Convegno internazionale di studi, Napoli 20-24 novembre 2001, éds. Paolo Lucentini, Ilaria Parri et Vittoria Perrone Compagni, Brepols, 2003, p. 491-503.

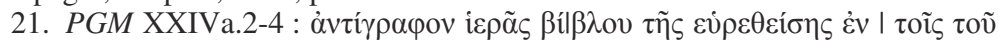

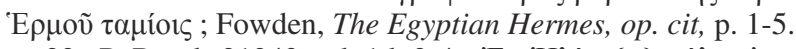

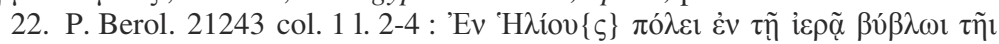

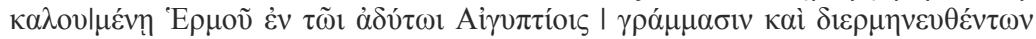

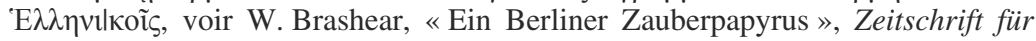
Papyrologie und Epigraphik [ZPE], 33, 1979, p. 261-278. 
de l'hermétisme ${ }^{23}$, la culture religieuse pharaonique ne devientelle pas hermétique dans son ensemble ? De la même manière, au Thot Trismégiste d'Hermopolis Magna, Fowden ajoute le Thot Paotnouphis de Pselchis (un illustre mégistos) ${ }^{24}$. Il les identifie presque l'un à l'autre, donnant ainsi l'impression d'une uniformité dans les actualisations religieuses du dieu Thot alors que le dieu de Pselchis répond à une logique théologique et locale qui le rattache spécifiquement au mythe de la déesse lointaine ${ }^{25}$. Les dieux Thot et Chou y apparaissent avec une fonction que n'a pas le Trismégiste : protéger la royauté solaire en apaisant la déesse lointaine, ce qui permet le retour de la crue et la sauvegarde du pays.

À l'issue de cette première section, deux questions doivent désormais guider l'enquête : a) quelle est la relation entre l'hermétisme et la magie (problème de la définition maximale) ? et b) comment circule le nom divin d'Hermès Trismégiste (problème de la définition minimale) ? En effet, si nous prenons le nom comme pierre de touche, il ne peut y avoir de Trismégiste sans hermétisme. Mais si l'on examine le contexte d'énonciation de certains documents, on est amené à se demander ce qui relie les différentes occurrences et comment définir ce lien.

\section{DES TRISMÉGISTES SANS HERMÉTISME}

\section{Mythologie et cultes locaux}

Dans les textes les plus anciens, l'épithète est réservée à Hermès, sauf dans un texte du Corpus Hermeticum où l'adjectif $\tau$ í́ $\sigma \mu \varepsilon \gamma \alpha \varsigma$ s'applique à Platon et où Hermès est alors qualifié de manière hyperbolique de $\mu v \rho t o ́ \mu \varepsilon \gamma \alpha \varsigma^{26}$. La forme $\tau \rho i ́ \sigma \mu \varepsilon \gamma \alpha \varsigma$ conserve ici la valeur laudative caractéristique du style égyptien, dont elle

23. Youri Volokhine, «Le dieu Thot et la parole», Revue de l'Histoire des Religions [RHR], 221 / 2, 2004, p. 131-156 dresse le portrait de ce dieu de l'écriture qui patronne toute la culture égyptienne. Il faut évidemment y voir un legs pharaonique passé plus tard à l'hermétisme (p. 152-156).

24. Fowden, The Egyptian Hermes, op. cit., p. 27.

25. Danielle Inconnu-Bocquillon, Le mythe de la déesse lointaine à Philae, Le Caire, Institut français d'archéologie orientale, 2001 et « Thot de Pnoubs (la ville) ou Thot de Nébès (l'arbre) », Revue d'Égyptologie [RdÉ], 39, 1988, p. 47-62.

26. Corpus Hermeticum XXI, 19. 
est issue, sans dénoter par elle-même l'idée d'hermétisme. C'est également le cas dans un emploi laudatif postérieur, où l'adjectif $\tau \rho \iota \sigma \mu \varepsilon ́ \gamma ı \sigma \tau o \varsigma$ est utilisé pour faire l'éloge de l'empereur Tibère II Constantin (578-582 $)^{27}$. Ce calque de l'égyptien vers le grec atténue quelque peu la valeur de nom propre que Festugière accordait à l'épithète et lui redonne celle qu'elle avait en égyptien et qu'elle a longtemps conservée, à savoir une valeur laudative d'exaltation que l'on retrouvera régulièrement dans des textes byzantins ${ }^{28}$.

La documentation papyrologique comporte d'autres attestations qui renvoient certes spécifiquement au Thot d'Hermopolis Magna mais sans connotation d'hermétisme. Le dieu donne son nom à des lieux-dits dans la division du territoire en propriétés et apparaît dans des contrats ${ }^{29}$. Dans une correspondance officielle du IV siècle entre la cité d'Hermopolis et le concitoyen Aurélios Ploution, proche de l'empereur Gallien, le conseil de la cité formule le vœu final que la divinité locale, "Hermès Trismégiste », offre un retour sans tempête à leur concitoyen ${ }^{30}$. Ce texte présente des difficultés non négligeables. Récemment, Anna van den Kerchove a tiré argument d'un rapprochement avec $C H$ I.2 pour défendre une parenté littéraire entre cette lettre et le corpus hermétique ${ }^{31}$. Une expression très similaire se retrouve dans les deux textes: $\pi \alpha \rho i ́ \sigma \tau \alpha \tau \alpha i ́$ бor

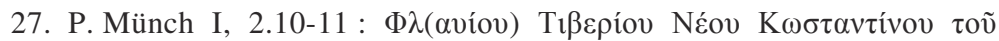

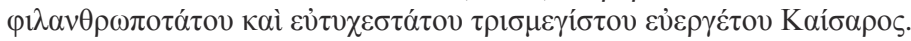

28. E. g. Theosophorum Graecorum Fragmenta (thesauri minores) $\Delta, 3$, éd. Erbse ('O $\Delta \tilde{\omega} v$ ó $\tau \rho 1 \sigma \mu \varepsilon ́ \gamma 1 \sigma \tau o \varsigma)$; Vie de Saint Luc le stylite 10, 31 (éd. Delehaye,

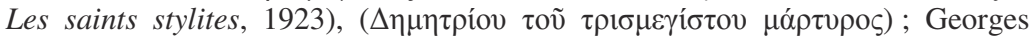
Pisidès, Heraclias I, 9 (éd. Pertusi, Giorgio di Pisidia. Poemi I. Panegirici epici,

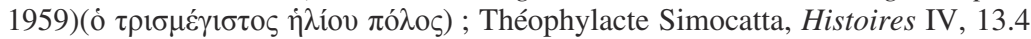

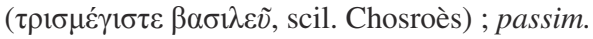

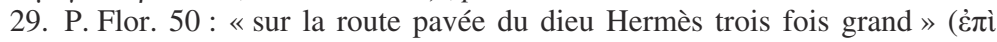

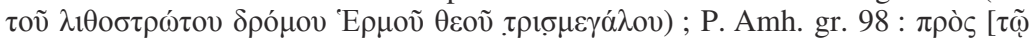

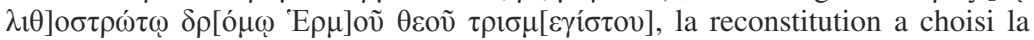
forme superlative mais on pourrait restituer peut-être plus légitimement la forme $\tau \rho \iota \sigma \mu \varepsilon \gamma \alpha \dot{\lambda}$ ov par comparaison avec le premier texte ; Papiri greci e latini XIV,

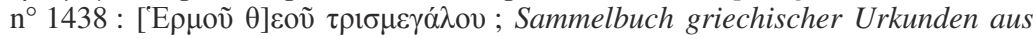

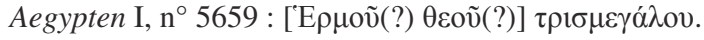

30. Studien zur Palaeographie und Papyruskunde XX, $\mathrm{n}^{\circ} 61$ (= P. Vind. G 12563) : « le dieu de nos ancêtres, Hermès trois fois très grand, qui t'assiste en

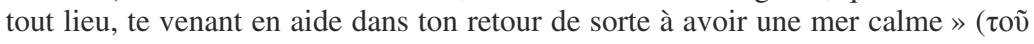

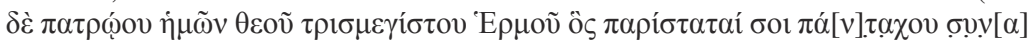

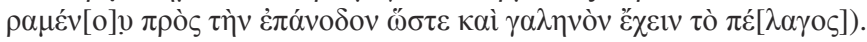

31. Anna Van den Kerchove, «Papyrological Hermetica », Studi e materiali di storia delle religioni, 83 / 1, 2017, p. 111-112. 


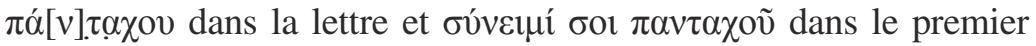
traité du corpus (où le sujet est Poimandres et où le destinataire est le narrateur). La lettre à Aurélios contiendrait ainsi une paraphrase du texte hermétique. Évidemment, une telle parenté est impossible à réfuter mais elle reste à mon avis du domaine de l'hypothèse. Dans quelle mesure peut-on faire dériver une formule de l'autre? Ne se peut-il pas que toutes deux soient une variation sur un thème commun ? Je signalerai donc simplement que la littérature atteste d'autres emplois des mêmes termes, si bien qu'on ne peut exclure un réseau plus large d'usages et de références brodant, en des termes analogues, sur le motif de l'assistance divine universelle ${ }^{32}$. Ainsi, dans une lettre du $\mathrm{IV}^{\mathrm{e}}$ siècle à sa famille, un jeune malade exprime sa « reconnaissance envers la providence divine qui [leur] vient en aide partout et en toute chose ${ }^{33}$. Plus proche de la formule en question et du thème du voyage, l'empereur Julien, dans un dialogue mythologique, rapporte le discours d'Hélios à un jeune

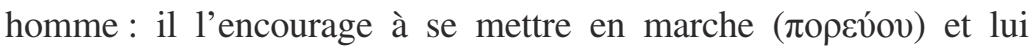
promet l'aide des dieux dans les termes de $\mathrm{CH}$ I. $.2^{34}$.

Si l'on se tourne maintenant vers la dimension locale, la lettre apparaît dans les archives de Théophane où l'on rencontre d'autres attestations du dieu égyptien de la cité dans les formules de salutation ${ }^{35}$. L'auteur semble vouer une forme particulière de

32. Chez Xénophon, Mémorables I, 1.19, les dieux, d'après Socrate, sont «présents partout » ( $\pi \alpha v \tau \alpha \chi 0 \tilde{v} \pi \alpha \rho \varepsilon \tilde{v} v \alpha \mathrm{l})$, omniprésence qui leur garantit l'omniscience (Mem. I, 4.18); chez les auteurs chrétiens, les variantes de l'expression sont très communes : Basile de Césarée, Hom. in illud: Attende tibi ipsi p. 34.13 (éd.

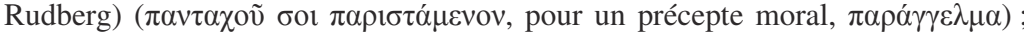
Jean Chrysostome, Patrologia Graeca Migne [MPG], LIV p. 499.3 (= In Genesim)

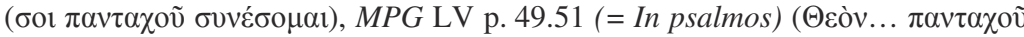

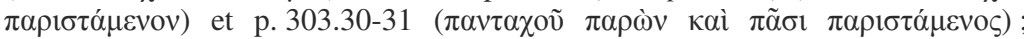
etc. Pour une problématique analogue concernant l'orphisme ou la philosophie populaire, voir Arthur Darby Nock, « Orphism or Popular Philosophy? », The Harvard Theological Review 33 / 4, 1940, p. 301-315.

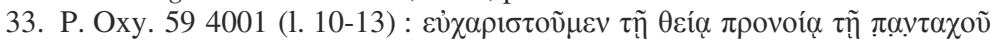

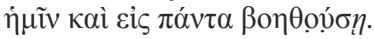

34. Julien, Contre Héraclius 22, 195-197 : «nous t'assisterons partout, moi,

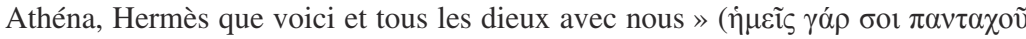

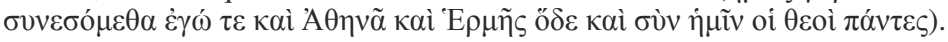

35. P. Herm 3 : «que te rendent heureux pour toujours Hermès lui-même, le

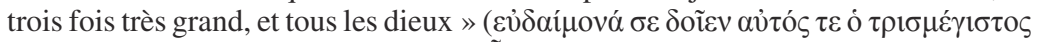

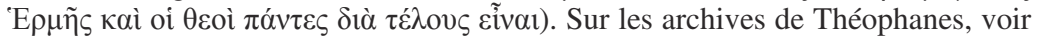
John Matthews, The journey of Theophanes : travel, business, and daily life in the Roman east, New Haven, Yale University Press, 2006, partic. p. 12-20. 
« dévotion au dieu Hermès protecteur $»^{36}$, mais en faire un « hermétiste » me paraît incertain. Il s'agit de «la divinité ancestrale »

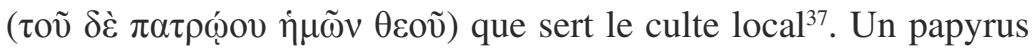
atteste d'ailleurs l'existence d'un lieu de culte dans la région d'Hermopolis : un sanctuaire dédié à Hermès trismégas apparaît dans la liste des temples consacrés à différentes divinités (Amon, Isis, "Athéna », c'est-à-dire Neith, Anubis, Souchos) ${ }^{38}$. S'il y a donc une communauté vénérant bel et bien Hermès Trismégiste, ce n'est pas celle que l'on attendrait, mais simplement celle de prêtres attachés au culte local d'une divinité ancienne parmi d'autres. Au demeurant, c'est un fait bien connu que le titre «trismégiste » est apparu dans un processus de traduction autour de la personnalité du Thot d'Hermopolis. Vers le $\mathrm{I}^{\mathrm{er}}$ ou le $\mathrm{II}^{\mathrm{e}}$ siècle, la forme $\omega \omega \omega$ a servi à rendre en grec la formule égyptienne de manière concurrente à l'épithète $\tau \rho \iota \sigma \mu \varepsilon ́ \gamma 1 \sigma \tau o \varsigma^{39}$. Des documents officiels ptolémaïques mentionnent également un Hermès deux fois grand ${ }^{40}$. La gémination de l'adjectif était une caractéristique stylistique égyptienne valant pour toutes sortes de dieux. Dans son dossier sur Hermès, Quaegebeur concluait que «Thot trois fois (très) grand ne se distingue donc pas vraiment de Thot deux fois (très) grand $\gg^{41}$. Dans ces circonstances, les dédicaces et les proscynèmes mentionnant le dieu dans les syringes et à Panopolis doivent-elles être prises comme des marques de l'hermétisme et de sa popularitét ${ }^{42}$ ? Si la référence spécifique au Thot

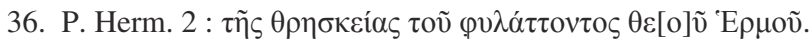

37. Fowden, The Egyptian Hermes, op. cit., p. 176 fait de Théophane et de son cercle «a group of learned devotees of Hermes ». Contra Robin Lane Fox, «Review of The Egyptian Hermes: A Historical Approach to the Late Pagan Mind », The Journal of Roman Studies, 80, 1990, p. 238.

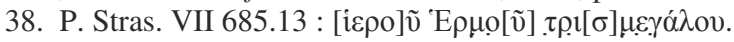

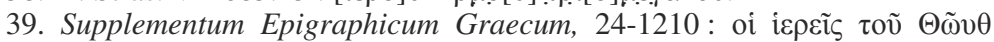
$\Omega \Omega \Omega$ Nò $\beta$ Z $\mu$ oṽv (= Mitt. des Deutschen Arch. Instituts, Abt. Kairo 20, 1965, $\mathrm{n}^{\circ} 121$; repris dans Étienne Bernand, Inscriptions grecques d'Hermoupolis Magna et de sa nécropole, $n^{\circ}$ 3, Institut Français d'Archéologie Orientale, 1999).

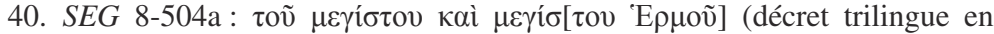
l'honneur de Ptolémée IV); Orientis Graeci Inscriptiones Selectae [OGIS],

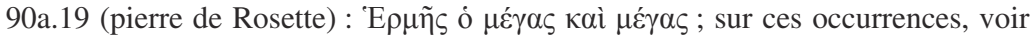
T. C. Skeat et E. G. Turner, "An Oracle of Hermes Trismegistos at Saqqâra », The Journal of Egyptian Archaeology [JEA] 54, 1968, p. 208.

41. Jan Quaegebeur, art. cit., p. 537.

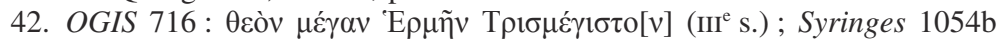

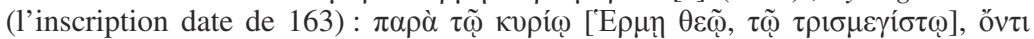

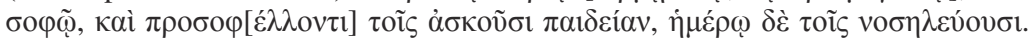


d'Hermopolis n'implique pas nécessairement l'hermétisme mais peut se comprendre dans le cadre cultuel égyptien, alors l'adjectif trismegas ou trismegistos dans les $P G M$ ne peut pas non plus être un gage d'hermétisme. Le nom du dieu est au contraire disponible pour des actualisations variées comme c'était le cas pour d'autres divinités locales populaires.

On trouvera également un Souchos Trismégiste dans le fameux hymne d'Isidore à Narmouthis ${ }^{43}$, lequel est le dieu crocodile du Fayoum servant, dans le contexte religieux de l'hymne, de sunnaos theos à la déesse Isis et identifié à l'agathos daimôn ${ }^{44}$. Il y apparaît

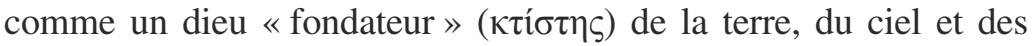
eaux. Cet ancien dieu égyptien prend en effet, à l'époque romaine, la dimension d'une divinité démiurgique primordiale, identifiée à de nombreux dieux solaires ${ }^{45}$. Divinité populaire du Fayoum, il est régulièrement accompagné du phénomène stylistique de gémination de l'adjectif $\mu \varepsilon^{\prime} \gamma \alpha \varsigma^{46}$. La triple répétition est aussi attestée pour les dieux crocodiles ses enfants dans une inscription du début du $\mathrm{II}^{\mathrm{e}}$ siècle av. J.-C ${ }^{47}$, et dans une inscription du Fayoum à un dieu « grand grand très haut » où on a voulu reconnaître le dieu juif, c'est probablement Souchos qui est le destinataire du texte ${ }^{48}$. Il se pourrait d'autre part que le Fayoum offre un ancrage local supplémentaire à

Malheureusement, dans cette inscription endommagée, le nom du dieu et son épithète semblent uniquement inférés à partir des informations qui suivent.

43. Étienne Bernand, Inscriptions métriques de l'Égypte gréco-romaine,

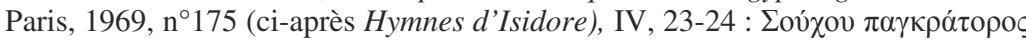

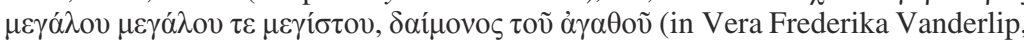
The four Greek Hymns of Isidorus and the Cult of Isis, Toronto, American Studies in Papyrology, 1972). I. S. Moyer, «Isidorus at the gates of the temple» in I. Rutherford (éd.), Graeco-Egyptian Interactions. Literature, Translation, and Culture, 500 BC-AD 300, Oxford, 2016, p. 209-244.

44. Voir Hymnes d'Isidore II.9-14 et les commentaires de Vanderlip, p. 38-39.

45. Lexikon der Ägyptologie [LdÄ], s. v. « Sobek», p. 1010.

46. Pour un relevé des sources, voir Alfred Twardecki, «Weihinschrift für Hermes oder Souchos ?», ZPE 99, 1993, p. 198 n. 4.

47. Étienne Bernand, Recueil des inscriptions grecques du Fayoum III.196:

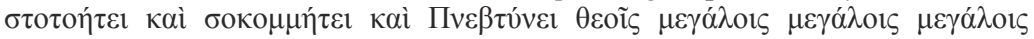

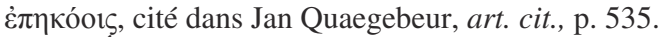

48. Étienne Bernand, Recueil des inscriptions grecques du Fayoum III.210:

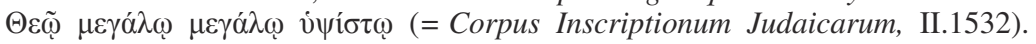
Contre l'attribution juive et pour l'hypothèse du dieu Sobek, voir Twardecki, art.

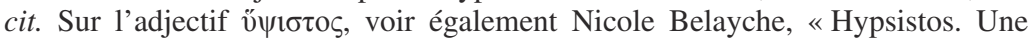
voie de l'exaltation des dieux dans le polythéisme gréco-romain », Archiv für Religionsgeschichte [ARG] 7, 2005, p. 34-55 (pour l'inscription en question, voir p. 39 n. 35). 
l'émergence de l'hermétisme : le nom de Poimandres, qui apparaît dans $\mathrm{CH} \mathrm{I}$, a donné lieu à plusieurs spéculations étymologiques pour le faire dériver soit du grec, soit du copte, avec le parti pris qu'il s'agissait d'une création ad hoc; mais on a avancé, il y a quelques années, la possibilité que ce nom serait le produit de transformations linguistiques à partir du nom du pharaon divinisé Amenemhet III, vénéré en particulier dans le Fayoum, et dont

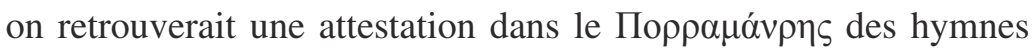
d'Isidore du temple de Narmouthis ${ }^{49}$. Le traité où apparaît le nom de Poimandres est tenu pour le plus ancien de ceux que nous avons conservés et ne mentionne pas le Trismégiste. Aussi s'agirait-il, pour H. Jackson, d'un traité proto-hermétique, composé dans le Fayoum par un dévot du pharaon divinisé ${ }^{50}$. En gagnant en prestige international, l'hermétisme aurait délaissé cette figure trop locale, qui ne pouvait rien évoquer à des lecteurs non-égyptiens, voire non originaires du Fayoum.

Dans le P. Leid. I 384, source démotique principale pour le Mythe de l'Eil du soleil, se trouve un Thot trois fois grand $\left(s_{3} \varepsilon_{3} \varepsilon_{3} D h w t\right)^{51}$ qui n'a, là aussi, rien d'hermétique mais tout de mythologique : il s'agit de fables animalières où le dieu a pris la forme d'un singe pour apaiser la déesse lointaine et le passage remplit une fonction étiologique à l'égard de la fête de Thot. La traduction de ce papyrus en grec, bien qu'incomplète, mentionne bien Hermès mais ne laisse

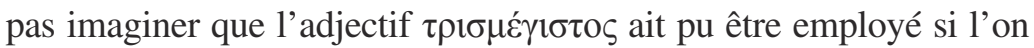
voit dans son emploi une référence à l'hermétisme tel que nous le décrivent les sources grecques et latines ${ }^{52}$. Le Thot trois fois grand

49. Howard M. Jackson, «A New Proposal for the Origin of the Hermetic God Poimandres », ZPE 128, 1999, p. 95-106 (notamment p. 105 pour l'explication linguistique). Hymnes d'Isidore IV, 34.

50. ibid. p. 105.

51. P. Leid. I 384, 21.32 et 22.11. Pour l'édition et la traduction du texte démotique, Françoise de Cenival, Le Mythe de l'oeil du soleil, Sommerhausen, G. Zauzich, 1988. Voir aussi J. Gwyn Griffiths, «Remarks on the Mythology of the Eyes of Horus », Chronique d'Égypte 33 / 66, 1958, p. 182-193.

52. Pour la version grecque (II - III ${ }^{\mathrm{e}}$ siècle), Stephanie West, «The Greek Version of the Legend of Tefnut », JEA 55, 1969, p. 161-183. Voir aussi Luigi Prada, «For a New Edition of P. Lond. Lit 192 : Current Research on the Greek Version of the Myth of the Sun's Eye », Actes du 26 congrès international de papyrologie, Genève, 16-21 août 2010, Genève, Droz, 2012, p. 627-634. Monica Signoretti, «A Tale of Two Tongues? The Myth of the Sun's Eye and its Greek Translation », in Proceedings of the Twenty-Fifth International Congress of Papyrology, Ann Arbor 2007, éds. Traianos Gagos et Adam Hyatt, 2010, p. 725-732 compare le texte démotique et 
est bel et bien présent dans le texte démotique, il est absent du texte grec. En revanche, un fait qu'il faudra garder à l'esprit lorsqu'il s'agira de considérer les Hermès des $P G M$, c'est que ce récit démotique, qui nous est conservé sur ce seul papyrus, est écrit au recto du PGM XII (lequel appartient à la collection d'Anastasi) ${ }^{53}$ ! Le rapprochement entre le corpus magique gréco-égyptien et la mythologie du dieu Thot dans ce document est donc incontournable.

L'ouverture du PGM IV contient par ailleurs un texte copte mentionnant «Thot deux fois grand et sage » dans une salutation aux dieux ${ }^{54}$, mais l'hermétisme n'est pas la catégorie la plus pertinente pour expliquer cette présence: la rhétorique d'éloge consistant à multiplier l'adjectif «grand» est bien attestée en égyptien et se retrouve pour d'autres dieux dans les $P G M$. On n'y rencontrera $\tau \rho \iota \sigma \mu \varepsilon^{\prime} \gamma 1 \sigma \tau o \zeta$ qu'une seule fois ${ }^{55}$. Le dieu apparaît alors dans sa fonction traditionnelle de scribe, dépositaire d'un savoir qu'il met par écrit sur les murs d'un temple. Ce savoir n'est pas une révélation d'ordre philosophique ou cosmologique mais d'ordre rituel : il est la transmission de paroles efficaces, qui sont les onomata du dieu et qu'il faut prononcer. Ici, le dieu en question est Osiris dont le praticien va énoncer des noms multiples formés par composition avec d'autres êtres divins.

La découverte toute récente, puis la publication et la traduction d'un Livre de Thot écrit en démotique sont venues ajouter une pièce précieuse au dossier de l'hermétisme dans la perspective du comparatisme interculturel ${ }^{56}$ : alors que faute d'antécédents

sa traduction grecque et pense que la version grecque a opéré des adaptations de la théologie égyptienne en fonction des références de son public hellénophone. Contra Prada, art. cit. p. 631 souligne la popularité du mythe et la diversité des versions démotiques qui ont dû circuler, ce qui oblige à une grande prudence dès qu'il s'agit d'évaluer les transformations lors du processus de traduction.

53. Sur les rapports entre le Mythe et le corpus magique, voir Françoise de Cenival, "Remarques sur le vocabulaire du "Mythe de l'œil du soleil" ", Aspects of Demotic Lexicography. Acts of the Second International Conference for Demotic Studies, éd. S. P. Vleeming, Louvain, Peeters, 1987, p. 3-8 et Korshi Dosoo, Rituals of Apparition in the Theban Magical Library, Thèse de doctorat, Macquarie University, 2014, p. 141-143.

54. PGM IV.19-20.

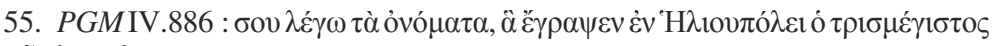

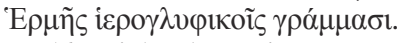

56. Richard Lewis Jasnow et Karl-Theodor Zauzich, Conversations in the house of life : a new translation of the ancient Egyptian Book of Thoth, Wiesbaden, Harrassowitz Verlag, 2014 (=Book of Thoth), qui reprend la première édition de 
incontestables, Mahé avait cherché dans la littérature de sagesse égyptienne les prémices du dialogue hermétique ${ }^{57}$, le Livre de Thot fournit le prototype attendu d'un enseignement transmis sous la forme d'un dialogue entre un maître et son élève tout en mentionnant le nom de Thot «trois fois grand» $(w r w r w r)^{58}$. Notons que le formulaire est original et ne correspond à aucune des formes mentionnées par J. Quaegebeur. Cette épithète et la ressemblance générique et structurelle de ce texte avec les hermetica ont ouvert la voie à la comparaison et ont donné de la matière pour défendre l'ancrage social égyptien de l'hermétisme ; mais tous les commentateurs restent prudents tant l'écart du contenu reste important entre les documents grecs et le texte démotique $^{59}$. On remarquera également que l'apprentissage prend ici la forme spécifique d'une leçon de géographie sacrée portant sur l'Égypte et se trouve en lien direct avec les activités scribales du clergé et du dieu Thot. Certaines influences du grec vers l'égyptien sont aussi à déceler dans ce texte où l'élève reçoit le nom de $m r r h$, qui serait la traduction littérale du terme $\varphi \imath \lambda$ ó

La plus ancienne attestation du Trismégiste en grec nous permettra d'en arriver au PGM VII. Il s'agit d'ostraca signés par un certain Horus, pastophore d'Isis, et retrouvés à Saqqarah ${ }^{61}$.

2005 : The Ancient Egyptian Book of Thoth : a demotic discourse on knowledge and pendant to the classical hermetica, Wiesbaden, Harrassowitz.

57. Mahé, Hermès en Haute-Égypte II. Le fragment du «Discours parfait » et les "Définitions » hermétiques arméniennes (NH VI, 8.8a), Québec, Presses de l'Université Laval, 1982, p. 279-305 et p. 449-456. Contra Fowden, The Egyptian Hermes, op. cit., p. 68-74.

58. Book of Thoth 1. 426 ; Jean-Pierre Mahé, «Preliminary Remarks on the Demotic "Book of Thoth" and the Greek Hermetica », Vigiliae Christianae 50 / 4, 1996, p. 353-363, en partic. p. 358-360, établit une liste de points d'analogie pour appuyer l'idée d'une parenté. Le nom d'Imhotep (= Asclépius) est également récurrent ; le Livre de Thot aurait à l'origine été conçu pour servir dans les drames lors du festival d'Imhotep, ibid. p. 361.

59. Jasnow et Zauzich, Book of Thoth, p. 49-50; Joachim Friedrich Quack, «Die Initiation zum Schreiberberuf im Alten Ägypten», Studien zur Altägyptischen Kultur 36, 2007, p. 260-261 ; Ghislaine Widmer, "Review of The Ancient Egyptian Book of Thoth », Journal of Near Eastern Studies 70 / 1, 2011, p. 113-116; Kevin van Bladel, « Review of : The Ancient Egyptian Book of Thoth », Bryn Mawr Classical Review, mai 2006.

60. Sur ce terme, voir Jasnow et Zauzich, The Ancient Egyptian Book of Thoth..., 2005, p. 13-15 et p. 511.

61. T. C. Skeat et E. G. Turner, «An Oracle of Hermes Trismegistos at Saqqâra », JEA 54, 1968, p. 199-208. 
Ce sont des brouillons pour un oracle rendu par Hermès trois

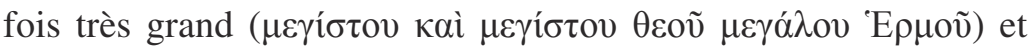
destiné aux trois dirigeants lagides régnant entre 170 et $164^{62}$. À l'évidence, leur auteur ne maîtrisait pas le grec. Le texte existe en plusieurs versions, dont la plus complète (peut-être la version finale ?) élimine inexplicablement la référence au dieu $^{63}$. Ces documents constituent le témoignage le plus ancien pour la traduction du triple adjectif égyptien en grec mais n'ont assurément rien d'hermétique à proprement parler : les procédures d'oracle en songe remonte au moins à l'époque ramesside et bien d'autres dieux peuvent être qualifiés pour la révélation ${ }^{64}$. Aussi faut-il ramener cette attestation aux pratiques culturelles courantes en Égypte pour l'envisager dans son milieu plutôt que d'y voir la genèse de l'hermétisme: l'apparition de l'épithète n'est pas l'apparition de l'hermétisme; l'hermétisme apparaît dans un «bain» culturel dont il finira par s'autonomiser sous l'effet de facteurs sociaux déterminés.

\section{La mise en rituel et l'Hermès Trismégas du PGM VII.540-578}

La procédure rituelle permettant d'obtenir l'oracle de Saqqarah ne nous est pas conservée. En revanche, le PGM VII.540-578 où figure Hermès trismégas donne des informations très riches sur son dispositif rituel. À la fin de l'Antiquité, dans un contexte de transition linguistique en situation interculturelle, les « archives d'Hermonthis » mentionnent le dieu Hermès en lui ajoutant l'épithète $\tau \rho ı \mu \varepsilon_{\gamma} \alpha \varsigma$ (PGM VII.551). Le papyrus date du $\mathrm{III}^{\mathrm{e}}$ ou du $\mathrm{IV}^{\mathrm{e}}$ siècle et provient de la Thébaïde - dans la région donc donc de Thèbes (d'où proviennent les papyrus de la «bibliothèque d'Anastasi ») et non loin de Nag Hammadi (dont le codex VI contient des textes hermétiques ${ }^{65}$ : en longeant le Nil, c'est une

62. ibid. p. 206.

63. ibid. p. 204.

64. Kasia Szpakowska, «The Open Portal. Dreams and Divine Power in Pharaonic Egypt », in Prayer, magic, and the stars in the ancient and late antique world, éds. Joel Thomas Walker, Brannon Wheeler et Scott B. Noegel, University Park (Pa.), Pennsylvania State University Pr., 2003, p. 111-124.

65. Concernant la «bibliothèque thébaine », voir Korshi Dosoo, «A History of the Theban Magical Library », Bulletin of the American Society of Papyrologists 53,2016 , p. 251-274 (p. 270 pour un tableau des textes retenus selon les auteurs). 
centaine de kilomètres qui sépare les trois sites. Le $P G M$ VII fait partie d'un ensemble comportant le $P G M$ VIII et le PGM XIa ; ce dernier est écrit sur le dos d'un document de comptabilité relatif à un grand domaine thébain, soit un domaine privé, soit celui d'un temple ${ }^{66}$. Le rituel des lignes 540-578 du PGM VII est généralement cité pour l'épithète d'Hermès en guise de preuve d'hermétisme à l'intérieur des $P G M$ : Hopfner le premier y décèle la trace de l'hermétisme ${ }^{67}$; Festugière, qui voit dans l'adjectif Trismégiste un "nom propre », le donne comme l'une des deux seules attestations en dehors du Corpus Hermeticum ${ }^{68}$; Fowden s'appuie également sur ce passage quand il inscrit la magie grécoégyptienne dans la sphère de l'hermétisme ${ }^{69}$; Betz a quant à lui émis l'hypothèse que les $P G M$ contiendraient un hermétisme en devenir, encore latent, et qu'ils en représenteraient donc une forme antérieure ${ }^{70}$.

Dans sa typologie des textes de magie hermétique, Festugière traduit ce rituel en retenant l'apparition d'Hermès comme son élément central ${ }^{71}$. Je montrerai qu'il n'occupe en réalité qu'un rôle secondaire et qu'il est invoqué pour favoriser l'apparition du dieu solaire. Le titre et le début de la praxis permettent de classer ce rite dans la catégorie des lychnomancies ${ }^{72}$ : le ritualiste doit placer une lampe dans une salle à l'est puis faire une offrande d'encens ${ }^{73}$ mais l'agent de la révélation est un «enfant

66. Korshi Dosoo, Rituals of Apparition in the Theban Magical Library, Thèse de doctorat, Macquarie University, 2014, p. 165-166.

67. Theodor Hopfner, Griechisch-ägyptischer Offenbarungszauber II, Leipzig, Haessel, 1921, § 220-221.

68. Festugière, op. cit., p. 74. L'autre occurrence: OGIS 716 (inscription votive d'un soldat à Pannopolis).

69. Fowden, The Egyptian Hermes, op. cit., p. 25-27.

70. H. D. Betz, The «Mithras Liturgy» : text, translation, and commentary, Tübingen, Mohr Siebeck, 2003, p. 37-38.

71. Festugière, op. cit., p. 289-290. Pour une typologie mise à jour, voir Emilio Suárez de la Torre, « Hermes en los papiros griegos de contenido mágico », in Que los dioses nos escuchen. Comunicación con lo divino en el mundo grecolatino y su pervivencia, Valladolid, Universidad de Valladolid, 2012, p. 275-289. Voir aussi Monaca Mariangelaca, «Ermete e la divinazione nei Papyri Graecae Magicae », art. cit.

72. Sur les lychnomancies, voir Athanassia Zografou, « Magic Lamps, Luminous Dreams. Lamps in PGM Recipes », Light and darkness in ancient Greek myth and religion, éds. Menelaos Christopoulos, E. D. Karakanza \& Olga Levaniouk, Lexington books, 2010, p. 276-294.

73. PGM VII.540-544. 
non-corrompu, pur $\gg^{74}$ qui sert d'intermédiaire. Voici le texte du logos à prononcer :

«Phisio iaô ageanouma skabarô skasabrôsou asabrô, parce que je vous demande en ce jour, en cette heure qu' apparaissent à cet enfant la lumière et le soleil, mane Ouseiri, mane Isi, Anubis, serviteur de tous les dieux, et fais que l'enfant entre en transe et qu'il voit les dieux, tous les dieux présents à la divination.Apparais-moi dans la divination, ô dieu à l'esprit élevé, Hermès trois fois grand ( $\tau \rho ı \mu \tilde{\varepsilon} \gamma \alpha \varsigma)$. Qu'apparaisse le dieu qui fit les quatre parties du ciel et les quatre fondations de la terre, resennèethô baseneraipan thalthachththachôthch chinebôth chinebôth mimylôth masynttori asobi. Viens à moi, toi qui es dans le ciel, viens à moi, toi qui sors de l'œuf. Je vous conjure au nom de entô tapsati legènisthô èlegè serphouth mouisrô lege [...] les deux dieux qui t'accompagnent, Thot. Le premier s'appelle Sô, le second Aph, kalou kagôèi sesophèi bainchôôôch » ${ }^{75}$.

Deux apparitions simultanées semblent se produire, l'une pour l'enfant, l'autre pour le ritualiste, chacune avec une invocation. Dans la première, le magicien s'adresse à un groupe de divinités qui remplissent une fonction médiatrice dans l'apparition indirecte puisqu'elles ont à charge les visions du médium. Le contenu de la vision attendue est explicité avec les sujets de la proposition infinitive à la fin de la phrase : la lumière, le soleil et Anubis. Dans la mesure où le groupe «mane Oseiri mane Isi » est inséré entre les premiers accusatifs et $\tau$ òv 'Avovßıv, il fait probablement partie du groupe sujet. Mane signifiant «bien-aimé » en égyptien, nous pouvons comprendre l'ensemble comme «le bien-aimé d'Osiris, le bien-aimé d'Isis $»^{76}$. S'agit-il de leur fils Horus, le dieu solaire? Ce ne serait pas surprenant à côté des mots $\varphi \tilde{\omega} \varsigma$ et

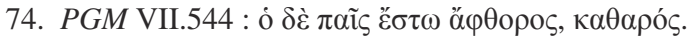

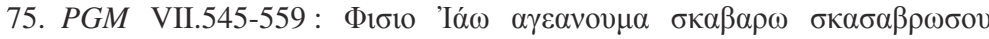

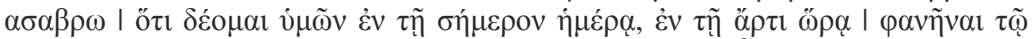

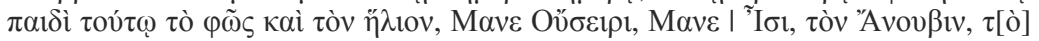

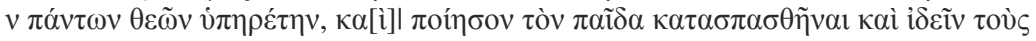

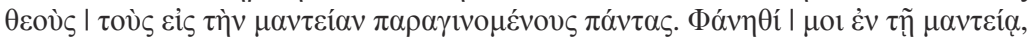

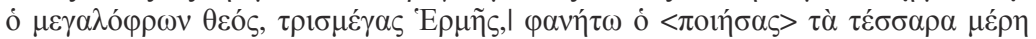

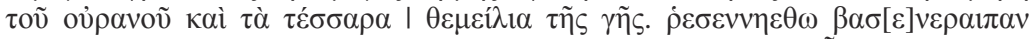

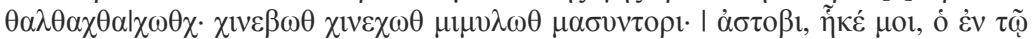

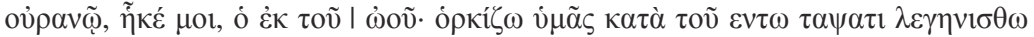

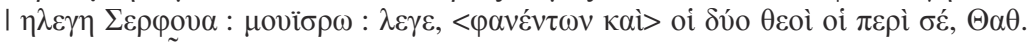

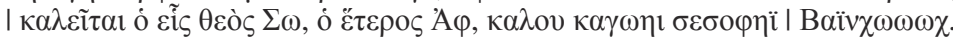

76. W. M. Brashear, «The Greek Magical Papyri : an Introduction and Survey », Aufstieg und Niedergang der römischen Welt, II.18.5, 1995, p. 3591 : de

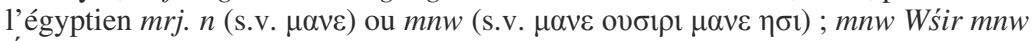
$S^{\prime} t=$ fils d'Osiris et d'Isis. 
en tout cas se lit plus loin dans une chaîne de noms barbares sous la forme "Harennôthès $»^{77}$. Un autre indice permet de souligner l'importance du dieu solaire dans ce rituel; il s'agit du trigramme solaire : serphouth mouisrô, qui signifie «Lotus-Lion-Bélier», symboles qui désignent les trois moments du cycle solaire quotidien: le lever, le zénith et le coucher ${ }^{78}$. Le contenu et le lieu de la révélation ne laissent donc pas d'ambiguïté. Et l'enfant lui-même est supposé en rendre compte : «Je vois ton seigneur dans la lumière ${ }^{79}$ devra-t-il dire au moment de l'apparition. La modalité de cette présence est par ailleurs conçue sur le modèle de l'impression et de la possession : un «esprit aérien » ( $\pi v \varepsilon v \tilde{\mu} \mu \alpha$ $\dot{\alpha} \varepsilon \rho \circ \pi \varepsilon \tau \dot{\varepsilon} \varsigma)$ doit «entrer dans son âme» pour lui permettre «d'imprimer ( $\tau 0 \pi \omega ́ \sigma \eta \tau \alpha l)$ la forme immortelle dans la lumière puissante et incorruptible $»^{80}$.

La seconde invocation commence avec $\varphi$ óv $\theta \mathrm{r}$ et contient une variation stylistique dans l'usage des déictiques singuliers et pluriels qui obscurcit également sa compréhension. En dépit de ce flottement, il reste clair qu'intervient dans le rituel une puissance cosmique. Créateur de l'espace céleste aussi bien que terrestre, le dieu qui apparaît est associé à l'œuf originel et c'est cette dimension originelle qui le qualifie, à l'évidence, pour exercer un rôle divinatoire. Loin d'être le dieu qui se révèle, Thot sert ici à son tour de médiation permettant d'introduire la puissance solaire. La mention d'un œuf d'où émerge un dieu rappelle par ailleurs la cosmologie d'Hermopolis dont Thot est le dieu majeur : l'ibis divin (ou une oie céleste) dépose un œuf sur la butte qui émerge de l'océan primordial (le Noun) ${ }^{81}$. Dans la même ligne, le mot

77. PGM VII.573. Harrenôthès ou Harendotès provient de l'égyptien $H r-n \underline{d}-j t$. $f$ et se traduit par « Horus qui prend soin de son père ». Le titre véhicule une idée de piété filiale dans l'accomplissement des devoirs envers le père défunt et l'héritage de ses fonctions ( $L d \ddot{A}$, s.v. «Harendotes »); dieu royal et solaire, Harendotès exprime à la fois la succession dynastique et la garantie de la résurrection d'Osiris. À Dakka, en Nubie, dans le temple de Thot, ce dieu est par ailleurs qualifié de « fils d'Isis, enfant d'Osiris » (Danielle Inconnu-Bocquillon, op. cit., p. 114, inscription 205).

78. Marie-Louise Ryhiner, «À propos de trigrammes panthéistes », RdÉ 29, 1977, p. 125-137.

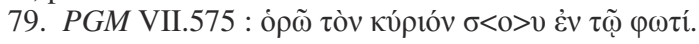

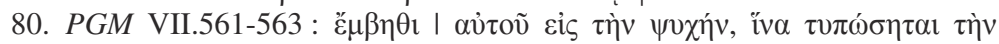

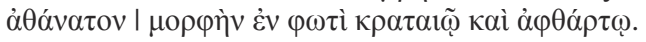

81. Serge Sauneron et Jean Yoyotte, «La naissance du monde selon l'Égypte ancienne », in La naissance du monde, Paris, Éditions du Seuil, 1959, p. 59-62. 


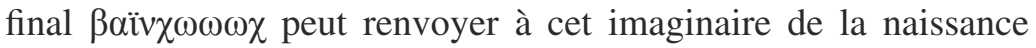
de la lumière hors des ténèbres originelles. Il signifie "âme des ténèbres » $\left(b_{3} n k k w\right)$ et apparaît régulièrement dans les $P G M$ et les defixiones $^{82}$. Une variante donne également la forme $\beta \alpha i v \varphi v o v v$, le $b a$ du Noun ${ }^{83}$, dans un papyrus dont la principale particularité est d'inscrire le rituel dans le cadre d'une cosmopoia reprenant des éléments égyptiens de la cosmogonie. Cette mythologie hermopolitaine de l'œuf convient parfaitement au rituel puisque le mythe raconte l'apparition de la lumière, premier événement du monde qui doit se reproduire maintenant pour l'enfant servant de médium. Ce n'est pas Hermès-Thot que l'on cherche à rencontrer, c'est le dieu solaire, mais on invoque le dieu Thot parce qu'il fait naitre le soleil. Aussi nous retrouvons-nous avec une figure d'Hermès/Thot dont la fonction dans le rituel dépend de son étroite relation avec la puissance lumineuse et créatrice qu'est le soleil. Cette association symbolique avec le soleil se trouvera dans une autre occurrence d'Hermès dans le PGM VII pour un charme de victoire consistant à écrire des charactères magiques sur une « lamelle solaire » ( $\left.\lambda \varepsilon \pi i ́ \delta \alpha \dot{\eta} \lambda \lambda_{1} \alpha \kappa \eta ̀ v, 1.919-920\right)$, c'est-à-dire en or.

Ce statut du dieu ne doit pas surprendre. Le dieu Thot apparaît dans l'imaginaire religieux égyptien comme une figure protectrice de la royauté solaire. Le mythe de la vache céleste en fait par exemple le vizir de Rér ${ }^{84}$. Dans le mythe de l'Eil de Ré, il a fonction d'intercesseur et, sous l'aspect de Chou, intervient en Nubie pour ramener la déesse lointaine (Hathor-Sekhmet) auprès de son père $(\text { Ré })^{85}$. Le mythe était très populaire et on en trouve de nombreuses allusions dans les Textes des pyramides ainsi que dans le Calendrier des jours fastes et néfastes ${ }^{86}$. Le fait que la copie démotique du mythe soit conservée avec le PGM XII de la «bibliothèque » thébaine est en soi éloquent. Il montre que

82. Brashear, art. cit., p. 3581.

83. PGM XIII.809.

84. Nadine Guilhou, "Myth of the Heavenly Cow», Encyclopedia of Egyptology, éds. Jacco Dieleman et Willeke Wendrich, University of California, Los Angeles, 2010, p. 4.

85. Voir supra n. 23.

86. Lana Troy, «Have a Nice Day! Some Reflections on the Calendar of Good and Bad Days », The Religion of the Ancient Egyptians. Cognitive Structures and Popular Expressions. Proceedings of Symposia in Uppsala and Bergen 1987 and 1988, éd. Gertie Englund, Uppsala, S. Academiae Ubsaliensis, 1989, p. $127-147$. 
ce mythe solaire égyptien nourrissait l'imaginaire de ceux qui possédaient les manuscrits de magie. Un Thot-Hermès trois fois grand dans le corpus magique est sûrement plus proche de ce Thot très populaire que du Trismégiste de l'hermétisme. Dans les $P G M$, Hermès pantocrator, associé au «cercle de la lune » (PGM

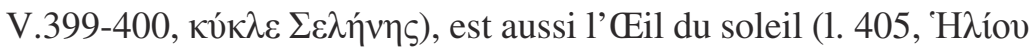
ó $\varphi \theta \alpha \lambda \mu \dot{\varepsilon})$ dans un hymne d'invocation que l'on prononce devant une statuette cultuelle du dieu au cours d'un rituel d'oniromancie. L'hymne se retrouve dans le PGM VII (1. 668-680) pour une autre oniromancie, mais une variation entre les deux mérite l'attention. La métaphore de l'œil disparaît cette fois au profit de celle du conducteur et Hermès devient «aurige du soleil» (1. 672-673:

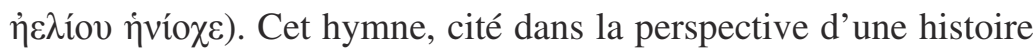
de l'hermétisme, constitue moins les prémices du mouvement philosophique que la continuation d'une pratique fort ancienne, où Thot n'a pas encore fonction de prophète, détenteur d'un savoir, mais celle de divinité, objet de vénération pour diverses fonctions qu'il occupe dans le monde, en particulier auprès du soleil. Cette mythologie solaire ne signifie pas que le dieu Thot n'apparait pas également dans le contexte des rituels divinatoires eu égard à d'autres de ses attributions. C'est par exemple le cas dans le rituel du PGM VII.335-347 : par son nom, il est associé à Chou ( $\Sigma \tilde{\omega} \varsigma)$ et par les modalités rituelles il intervient en tant que dieu de la connaissance.

Faire une histoire de l'hermétisme pose toujours de multiples difficultés. L'une d'elles est qu'en cherchant ses prémisses dans sa préhistoire gréco-égyptienne, on peut être tenté de considérer d'office l'épithète comme un "nom propre » ou de concevoir la magie comme une forme d'hermétisme. Cette catégorie n'épuise pourtant pas les manifestations d'Hermès Trismégiste. Divinité traditionnelle parmi d'autres, déjà distinguée en égyptien par la triple épithète dans le contexte cultuel d'Hermopolis, "Thot trois fois très grand, maître d'Hermopolis, le grand dieu » (D $\underline{h w} w$ tj $\varepsilon_{3} \varepsilon_{3} \varepsilon_{3} w r n b$ Hmnw p3 ntr $\left.\varepsilon_{3}\right)^{87}$ peut naturellement donner lieu à différentes actualisations lors des processus de traduction.

87. Jan Quaegebeur, art. cit. p. 533 : ce formulaire précis a été retrouvé en 1982, à Moscou, en démotique, et constitue la forme la plus complète et la plus proche de l'adjectif grec. L'inscription date de 105 av. J.-C. 
Le paradigme hermétique ne recouvre donc pas entièrement le «vieux» paradigme mythologique, de sorte qu'on pourrait certainement rencontrer d'autres emplois de l'épithète en démotique ou en grec sans avoir besoin de recourir aux concepts de l'hermétisme pour en rendre compte. Évidemment, la distinction que j'ai faite ici entre «magie» et « hermétisme » est heuristique et ne signifie pas que la voie d'Hermès n'incluait pas de pratiques rituelles et magiques. Inversement, les magiciens ont très bien pu puiser dans l'hermétisme ce qui leur convenait, ne serait-ce que le nom du dieu. Mais il est certainement difficile de mesurer précisément ces phénomènes. La distinction des deux catégories servait donc à interroger la communication entre ces deux champs et nos réflexes interprétatifs à la découverte d'une occurrence de l'adjectif. Ce qui apparaît, c'est que la diversité des occurrences n'est pas réductible à une catégorie de l'histoire de la philosophie, même si elles entretiennent avec elle des liens évidents.

\section{« L'HERMÉTISME », UNE STRATÉGIE POUR FAIRE IDENTITÉ ?}

Qu'il s'agisse de les dater ou de les situer dans une culture, un problème récurrent des hermetica grecs dans l'historiographie moderne a porté sur leurs origines. À la suite de plusieurs philologues du XvI ${ }^{\mathrm{e}}$ siècle, Isaac Casaubon réussit à prouver en 1614 qu'il s'agissait de textes tardifs alors qu' on les avait tenus pour la révélation de la prisca theologia des Anciens ${ }^{88}$. Au Xx ${ }^{\mathrm{e}}$ siècle, la question se pose de leur attribution culturelle et de leur ancrage social dans un milieu. Contre l'idée de confréries hermétiques défendue par Reitzenstein ${ }^{89}$ et l'opinion antique que les hermetica seraient des traductions de l'égyptien, Festugière a soutenu que ces textes ne relevaient pas d'une école ou d'une religion particulière

88. Casaubon, XVI Exercitationes De rebus sacris et ecclesiasticis ad Cardinalis Baronii Prolegomena in Annales. Pour l'historique des débats, voir Frederick Purnell Jr., «Francesco Patrizi and the critics of Hermes Trismegistus », in Das Ende des Hermetismus : historische Kritik und neue Naturphilosophie in der Spätrenaissance Dokumentation und Analyse der Debatte um die Datierung der hermetischen Schriften von Genebrard bis Casaubon (1567-1614), éd. Martin Mulsow, Tübingen, J. C. B. Mohr, P. Siebeck, 2002, p. 105-126.

89. Richard Reitzenstein, Poimandres, Studien zur griechisch-ägyptischen und frühchristlichen Literatur, Teubner, 1904. 
mais représentaient la combinaison de la philosophie hellénistique avec le stéréotype classique de la sagesse égyptienne ${ }^{90}$. Il s'agirait simplement d'une fiction «orientaliste » imposée sur des textes grecs. Aujourd'hui cependant, Mahé et Fowden ont insisté sur la mixité culturelle présente dans l'hermétisme en relevant les traces d'un fond égyptien bien réel, le premier en développant une approche littéraire de l'hermétisme et le second une approche historique $^{91}$. À leur suite, Copenhaver a fourni une nouvelle traduction des hermetica prenant acte d'un milieu gréco-égyptien pour la production de ces textes ${ }^{92}$. Avant eux, Derchain avait insisté sur l'irrécusable fond égyptien que l'on peut déceler dans les hermetica ${ }^{93}$. Aussi le caractère interculturel de l'hermétisme représente-t-il dorénavant un fondement solide pour la recherche. Ce sont les dynamiques de production qui sont plus difficiles à cerner. Cette dernière section s'attachera à souligner l'aspect politique de «l'hermétisme », à savoir la définition d'une identité religieuse collective au sein d'une société internationale. Elle traitera également de l'absence paradoxale de référence païenne à l'hermétisme avant Jamblique.

Il n'est certainement pas anodin qu'un des meilleurs témoignages que nous possédions sur l'élaboration de l'hermétisme antique se trouve chez Jamblique, alors même que les auteurs païns mentionnent moins souvent Hermès Trismégiste que les auteurs chrétiens ${ }^{94}$ : " les livres qui passent pour être d'Hermès contiennent bien, nous dit Jamblique, les doctrines enseignées par Hermès, même s'ils usent souvent du langage des philosophes

90. André-Jean Festugière, La révélation d'Hermès Trismégiste I, op. cit., p. 81-87.

91. Jean-Pierre Mahé, Hermès en Haute-Égypte I, op. cit., p. 22-26, 33-38 et Hermès en Haute-Égypte II, op. cit., p. 68-113, 278-308, 449-456; Fowden, The Egyptian Hermes, op. cit.

92. Brian P. Copenhaver, Hermetica : the Greek «Corpus Hermeticum» and the Latin "Asclepius » in a new English translation, with notes and introduction, Cambridge, Royaume-Uni, Cambridge University Press, 1992.

93. Ph-J. Derchain, «L'authenticité de l'inspiration égyptienne dans le "Corpus Hermeticum" », RHR $161 / 2$, 1962, p. 175-198. Voir aussi László Kákosy, «Hermes and Egypt », in A. B Lloyd et John Gwyn Griffiths (dirs.), Studies in pharaonic religion and society in honour of J. Gwyn Griffiths, London, Egypt Exploration Society, 1992, p. 258-261 et John Gwyn Griffiths, «Possible Egyptian Elements in Tractate XIII of the Corpus Hermeticum », Aspekte spätägyptischer Kultur, vol. 7, 1995, p. 97-102.

94. Kerchove, art. cit. p. 101-102. 
[grecs], parce qu'ils ont été traduits de l'égyptien par des hommes qui n'étaient pas dépourvus de culture philosophique $»^{95}$. Faut-il s'étonner qu'un auteur écrivant sous la couverture de l'Égypte, en la personne d'Abamôn, auteur factice de la réponse à Porphyre, soit si sensible à la transmission des savoirs égyptiens aux Grecs? Le montage littéraire qu'il établit dans le dispositif énonciatif, en inventant une figure d'auteur, crée une mise en abîme: le processus de création de l'hermétisme dans la traduction vers le grec se reflète dans la ré-attribution fictive d'une théologie néoplatonicienne à un Égyptien, garant de son authenticité. Si la dispute de Jamblique et de Porphyre intéresse l'hermétisme, ce n'est pas uniquement pour les témoignages qu'elle apporterait : les deux Syriens, l'un de Tyr, l'autre de Chalcis, illustrent également des options culturelles divergentes dans la construction d'une identité philosophique grecque ainsi qu'un rapport de l'universel et du local qui est inversés6. À l'analyse de leur nom - traduit pour l'un, transcrit pour l'autre - et de leur ville de naissance - une cité ouverte à l'international pour le premier, pour le second un bastion militaire enclavé dans un désert où se retireront les ermites Struck a montré que la querelle est, tout autant que philosophique, de nature sociale, et qu'elle est ancrée dans le rapport à l'espace : leur désaccord se situe dans un positionnement au sein d'une société polarisée entre universalisme et particularisme ${ }^{97}$. L'enjeu est en fait au niveau des rapports de l'hellénisme, en tant qu'universalisme culturel, avec les cultures religieuses locales. Les options philosophiques retenues constituent alors un parti-pris politique : valoriser la diversité des pratiques cultuelle dans l'accès au divin ou privilégier un idéal religieux commun fondé sur une raison philosophique. Cette divergence se lit également dans les modalités de la citation et des références à l'hermétisme : alors que Jamblique emploie le nom divin pour faire autorité, Porphyre se contenterait du gentilice substantivé «l’Égyptien» (ó Aîvó $\pi \tau \iota \varsigma \varsigma)$

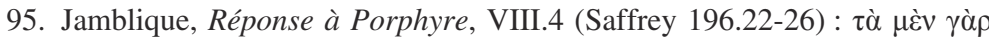

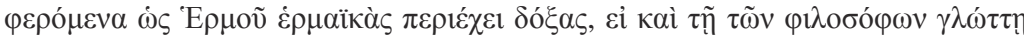

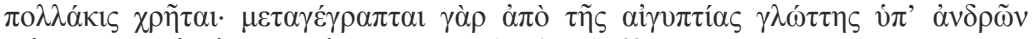
фı

96. Peter T. Struck, «Speech Acts and the Shakes of Hellenism in Late Antiquity », in Magic and ritual in the ancient world, éds. Marvin W. Meyer et Paul Allan Mirecki, Leiden, Pays-Bas, Brill, 2002, p. 387-403.

97. loc. cit. 
dans un passage du De abstinentia II, 47.198. La mention d'Hermès Trismégiste pourrait trahir une volonté de promouvoir une identité locale, ou du moins de trouver une stratégie énonciative pour affirmer une altérité, voire une supériorité. Dans le corpus hermeticum, on percevra notamment la compétition linguistique avec la faveur accordée à l'égyptien contre le grec : les traductions

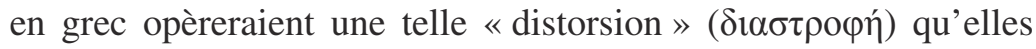

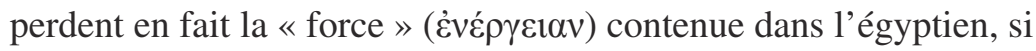

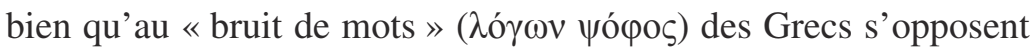

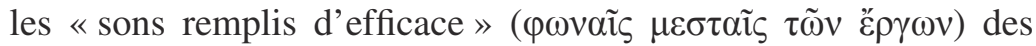
Égyptiens ${ }^{99}$. La méthode de Porphyre permet au contraire de niveler les différences dans une convergence universelle des doctrines.

À la lecture d'une autre occurrence de l'épithète, on comprendra à quel point les revendications identitaires ont pu être constitutives de l'hermétisme. Eusèbe fournit une attestation de l'adjectif $\tau \rho ı \sigma \mu ́ \gamma 1 \sigma \tau o \varsigma$ dans un texte qui pose de nombreux problèmes ${ }^{100}$. Le chapitre X du premier livre de la Préparation Évangélique expose la «théologie des Phéniciens » à partir de citations tirées de Philon de Byblos, qui se fonde lui-même sur un certain Sanchuniaton. Ces citations décrivent une théogonie où se mélange ce qui est authentiquement phénicien, ouvertement grec et subrepticement

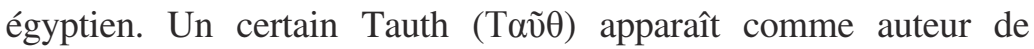
mémoires sur la création du monde ${ }^{101}$, laquelle raconte les rivalités entre Kronos et son père Ouranos et fait d'Hermès Trismégiste l'aide et le conseiller de Kronos ${ }^{102}$. Tauth ou Taaut est la forme phénicisée du dieu égyptien Thot $^{103}$; l'auteur des mémoires et l'adjuvant de Kronos seraient une seule personne mais le changement

98. André-Jean Festugière, «Une source hermétique de Porphyre : 1' "Égyptien" du De Abstinentia (II, 47) », Revue des Études Grecques 49/233, 1936, p. 586-595 a reconnu dans ce passage une allusion à l'hermétisme (sujette à caution).

99. Corpus Hermeticum, XVI, 1-2. Cf. Plotin, Traité 31 (V, 8) 6, où le philosophe reproduit l'image d'Épinal d'une écriture hiéroglyphique exprimant immédiatement l'intelligible.

100. Sur le texte de Philon, voir Albert I. Baumgarten, The "Phoenician history » of Philo of Byblos : a commentary, Leiden, Brill, 1981.

101. Eusèbe, Préparation Évangélique, I, 10.5.

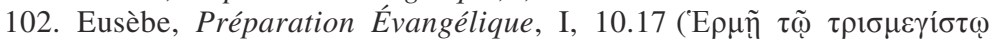

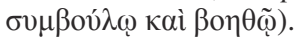

103. Baumgarten, op. cit. p. 72. 
de nom s'explique difficilement ${ }^{104}$. Ce récit «phénicien», dont l'auteur mythique est un Égyptien, est ensuite étroitement posé en équivalence avec le mythe de l'âge d'Or des Grecs ${ }^{105}$. Au contraire des autres textes mentionnant le Trismégiste chez les auteurs chrétiens, notamment Tertullien et Lactance, Hermès n'apparaît pas ici avec cette épithète comme le révélateur d'une sagesse - nulle trace des thématiques de l'hermétisme: c'est dans un univers mythologique plus traditionnel qu'il est introduit avec un rôle d'adjuvant habile. On lui reconnaîtra un aspect égyptien dans son association avec une divinité incarnant la justice et la souveraineté (Kronos), en opposition à un pouvoir désorganisateur (Ouranos), caractéristique majeure du dieu Thot. Sa fonction de secrétaire est étrange et il pourrait s'agir d'un emprunt à l'Égypte hellénistique ${ }^{106}$. Que cette occurrence chez le théologien chrétien provienne en réalité d'un auteur païen de la fin du $\mathrm{I}^{\mathrm{er}}$ siècle apr. J-C. est intéressant : elle témoigne que l'adjectif $\tau \rho \iota \mu \varepsilon^{\prime} \gamma \imath \tau \tau$ a pu circuler en grec dans des contextes non hermétiques mais caractérisés par des problématiques d'identité culturelle locale par rapport à l'identité internationale imposée par l'hellénisme. On devine en effet que, chez Philon de Byblos, la narration du mythe répond à une logique de rivalité avec les Grecs, qu'il accuse de s'être appropriés et d'avoir déformé, dans leur mythologie, des vérités phéniciennes ${ }^{107}$.

À défaut de fournir une réponse, ce détour vers Philon, Porphyre et Jamblique invite donc à un questionnement sur les facteurs sociaux qui conditionnent le développement de l'hermétisme en tant que catégorie religieuse et philosophique. Son émergence est contemporaine de l'apparition d'autres taxinomies, qui visent à supplanter des identités ethniques par une identité religieuse définie dans le rapport au divin ${ }^{108}$. De plus, il faut se souvenir qu'à partir de la fin du II $^{\mathrm{e}}$ siècle, la «caste » sacerdotale égyptienne, tout en connaissant une formidable production intellectuelle, a déjà perdu la majeure partie de son emprise économique sur les communautés

104. ibid.p. 192.

105. Eusèbe, Préparation Évangélique, I, 10.30.

106. Baumgarten, op. cit., p. 192. Pour Hermès (= Anubis) en conseiller d'Osiris, cf. Hécatée, fr. 25.137 in Felix Jacoby (éd.), Die Fragmente der griechischen Historiker vol. IIIa, Leyde, 1940.

107. Eusèbe, Préparation Évangélique, 10.40-41.

108. Francesco Massa, «Nommer et classer les religions aux II ${ }^{\mathrm{e}}-\mathrm{IV}^{\mathrm{e}}$ siècles : la taxinomie "paganisme, judaïsme, christianisme" », RHR 234 / 4, 2017, p. 689-715. 
locales du fait d'une fiscalité impériale favorisant les propriétaires terriens combinée à un fort contrôle de Rome sur la prêtrise ${ }^{109}$. Fin des travaux de constructions initiés par les premiers empereurs, transformation du temple de Thèbes en fort militaire au début du $\mathrm{IV}^{\mathrm{e}}$ siècle, édit du préfet en 199 pour interdire la divination et christianisation des campagnes et de l'empire referment la communauté des prêtres sur elle-même ${ }^{110}$. David Frankfurter a montré brillamment qu'il faut chercher dans cette évolution de la société la transformation des anciens prêtres en sorciers des villes et des villages d'Égypte ${ }^{111}$. C'est également à partir de là qu'il faut comprendre l'émergence d'un discours identitaire hermétique: une fois rompue l'alliance entre l'élite politique et le milieu sacerdotal, les prêtres sont contraints d'inventer une nouvelle manière d'exister et ne peuvent le faire que dans les termes et les conditions posées par l'étranger.

Le fait que ce sont surtout des auteurs chrétiens qui citent le nom d'Hermès Trismégiste est assez révélateur. Éventuellement, on peut en conclure que les auteurs païens sont si intimement connaisseurs de l'hermétisme qu'ils n'en citent pas l'autorité divine, tenue pour reconnaissable ${ }^{112}$. Mais il est également possible d'y voir un changement de paradigme dans la conception de l'autorité : si Hermès Trismégiste n'existe pas chez les auteurs païens, c'est qu'ils n'en ont tout simplement pas besoin car l'identité grecque ne forme pas un enjeu. Pour les apologistes chrétiens au contraire, il occupe une fonction importante: situer leur communauté religieuse par rapport à une collectivité polythéiste pour laquelle l'autorité réside justement dans la tradition et l'ancestralité. Hermès Trismégiste présente alors l'avantage de concilier deux critères d'autorité : l'Antiquité du savoir religieux (cruciale dans

109. Damien Agut-Labordère et Juan Carlos Moreno García, L'Égypte des pharaons : de Narmer à Dioclétien, 3150 av. J.-C.-284 apr. J.-C., Paris, Belin, 2016, p. 737-751.

110. ibid.p. 751-753 et p. 764-769. P. Yale inv. 299 (Sammelbuch griechischer Urkunden aus Aegypten XIV, ${ }^{\circ}$ 12144) pour la circulaire de 199 sur la divination et John Rea, «A New Version of P. Yale Inv. 299 », ZPE 27, 1977, p. 151-156.

111. David Frankfurter, Religion in Roman Egypt : assimilation and resistance, Princeton, Princeton University Press, 1998, p. 198-237 et « The Consequences of Hellenism in Late Antique Egypt : Religious Worlds and Actors », ARG 2 / 1, 2010, p. 162-194.

112. Kerchove, art. cit. p. 110-112 va en ce sens dans son analyse de la lettre d'Aurélios. 
le cadre polythéiste) et sa révélation divine (fondamentale pour les nouveaux courants religieux). En ce sens, ce nom divin incarne un pouvoir dans la construction d'une identité par rapport au champ religieux de l'Antiquité tardive.

Dès sa première apparition dans la littérature chrétienne, Hermès Trismégiste sert de témoin à charge contre l'évhémérisme chez Athénagoras ${ }^{113}$. Afin de défendre l'authenticité de la révélation chrétienne, Lactance mentionne Hermès Trismégiste à côté de la Sybille $^{114}$. Pour cette dernière, on sait qu'elle a été une pièce pratique dans la construction chrétienne d'une histoire de la révélation chez Augustin $^{115}$. De même, les citations d'Hermès Trismégiste offrent à Lactance «le visage païen de la pensée chrétienne ${ }^{116}$. Augustin retourne contre le polythéisme la prophétie d'Hermès dans l'Asclepius $^{117}$. Chez Cyrille d'Alexandrie, la référence à Hermès sert le même dessein polémique: convertir l'histoire profane à la révélation chrétienne et jouer une autorité païenne, apparentée à Moïse, contre la réforme de Julien « l'apostat ${ }^{118}$. Chez Didyme Caecus, Hermès Trismégiste permet de confirmer les interprétations de la Bible $^{119}$. Quant à Cosmas de Jérusalem au VIII ${ }^{\mathrm{e}}$ siècle, il intègre «Hermès trisaristos » à l'économie de la révélation et donne à l'épithète du dieu une connotation trinitaire ${ }^{120}$. À côté de ces réemplois, on trouvera aussi des attaques: Hippolyte mentionne le dieu dans une citation qu'il tire d'un écrit gnostique où il figure

113. Athénagoras, Supplicatio 28, 6.

114. Lactance, Institutions Divines, IV , 6.3, 6.9 ; II, 8.48.

115. Jean-Michel Roessli, "Augustin, les sibylles et les Oracles sibyllins. », Augustinus afer. Saint Augustin: Africanité et universalité. Actes du colloque international, Alger-Annaba, 1-7 Avril 2001, éds. Pierre-Yves Fux, Jean-Michel Roessli et Otto Wermelinger, Fribourg, Éditions Universitaires de Fribourg, 2003, p. 263-286.

116. Paolo Scarpi, «L'autorité divine d'Hermès Trismégiste, pour une "nouvelle religion de la tolérance" ", in Autorité des auteurs antiques : entre anonymat, masque et authenticité, éds. Maria Gorea et Michel Tardieu, Turnhout, Brepols, 2014, p. 105.

117. Asclepius 24 ; Augustin, Cité de Dieu, VIII, 23-27. Voir Pier Franco Beatrice, "Hermétisme», Encyclopédie Saint Augustin. La Méditerranée et l'Europe $I^{e}-X X I^{e}$ siècle, trad. Jean-Michel Roessli, éd. Marie-Anne Vannier, Éditions du Cerf, 2005, p. 695-697.

118. Cyrille, Contre Julien, I, 41.

119. Didyme Caecus, In Ecclesiasten p. 167 (Kramer); In psalmos p. 88 (Gronewald) ; De Trinitate III, 28.

120. Cosmas, In Gregorii Nazianzeni carmina, LXIV, 241-249, 1. 168-170 (éd. Lozza, Naples, M. D’Auria, 2000). 
parmi une liste d'auteurs (Ostanès, Pétosiris, Bérose, Zoroastre...) et où il incarne l'ignorance du divin ${ }^{121}$; Arnobe, quant à lui, se moque des sectateurs de Platon, de Pythagore ou d'Hermès ${ }^{122}$.

Dans les milieux juifs, d'autre part, Hermès est lui-même présenté assez tôt comme l'interpretatio de Moïse ${ }^{123}$, dont Musée, le maître d'Orphée, serait l'équivalent pour les Grecs ${ }^{124}$; Artapan (II ${ }^{\mathrm{e}} \mathrm{I}^{\mathrm{er}}$ siècle av. J.-C.) réécrit l'histoire de Moïse pour en faire le fondateur d'Hermopolis et le législateur de l'Égypte, pour l'associer à l'ibis, lui donner les attributs traditionnels de Thot et, en un mot, substituer l'autorité juive par excellence à la divinité égyptienne ${ }^{125}$. Il alimente par là «les traces de mémoire » constituant l'identité juive sur sa rupture avec l'Égypte ${ }^{126}$. Si l'on se tourne du côté de la papyrologie, on prendra la juste mesure de ce qu'ont pu être les rivalités identitaires dans la valorisation d'Hermès Trismégiste. Deux fragments hermétiques apparaissent dans P. Vind. G 29456 et 29828, dont le verso contient des fragments de l'apocryphe juif de Jannès et Jambrès, composé au tournant du $\mathrm{III}^{\mathrm{e}}$ siècle $^{127}$. Ces fragments, parmi les plus anciens, pourraient émaner d'une communauté judéo-chrétienne installée en Égypte ${ }^{128}$. Quant à Jannès et Jambrès, ce sont les deux «magiciens» de Pharaon auquel s'oppose Moïse. Enfin, le P. Leid. J 395 (PGM XIII) contient une longue cérémonie présentée sous plusieurs variantes et donnée comme le Huitième Livre de Moïse ${ }^{129}$. Alors qu'il évoque les équivalences

121. Hippolyte, Réfutation de toutes les hérésies, V, 14.8.

122. Arnobe, Contre les gentils, II, 13.

123. Eusèbe, Préparation Évangélique, IX, 27.6. Sur ce sujet, voir Gerard Mussies, «The Interpretatio Judaica of Thot-Hermes », in Matthieu Sybrand, Huibert Gerard, Heerma van Voss, Jan Zandee (dirs.), Studies in Egyptian religion : dedicated to Professor Jan Zandee, Leiden, E.J. Brill, 1982, p. 89-120.

124. Eusèbe, Préparation Évangélique, IX, 27.3-4.

125. Eusèbe, Préparation Évangélique, IX, 27 = Artapan fr. 3 dans Philippe Borgeaud, Thomas Römer, Youri Volokhine et Daniel Barbu (dirs.), Interprétations de Moïse. Égypte, Judée, Grèce et Rome, Leiden-Boston, Brill, 2010, p. 29-39 (édition et annotation du texte).

126. Voir Jan Assmann, Moïse l'Égyptien, Flammarion, 2010 (1997 pour l'édition allemande).

127. Kerchove, art. cit., p. 98-99, 104 ; The Apocryphon of Jannes and Jambres the Magicians, éd. Albert Pietersma, Leiden, New York, Köln, Brill, 1994.

128. Kerchove, art. cit., p. 108-109.

129. Sur ce document complexe, voir en particulier, pour sa structure et son histoire : Morton Smith, « The Eighth Book of Moses and how it grew (P. Leid. J 395) », Atti del XVII congresso internazionale di papirologia, vol. 2, Centro 
astrologiques entre sept types d'encens et les planètes, le copiste apporte une information précieuse : «Hermès, affirme-t-il, a volé les 7 fumigations dans ce livre et les a évoquées dans son propre livre sacré appelé l'Aile ${ }^{130}$. Ce document aide à percevoir que les rivalités interculturelles sont fondamentalement inscrites dans l'hermétisme. Qui plus est, le livre y apparaît comme le médium véhiculant la vérité, mais aussi l'identité et le conflit, comme pour les nouveaux courants religieux ${ }^{131}$. Aussi la fabrication d'Hermès Trismégiste en «prophète », auteur d'un texte contenant un savoir religieux, ressemble à une réponse aux nouveaux critères de piété s'imposant progressivement. Elle suppose une certaine proximité entre les milieux égyptiens traditionnels et les communautés juives ou chrétiennes vivant en Égypte, mais également la problématique d'une définition de soi dans une société multiculturelle ${ }^{132}$.

Ces rivalités n'ont pas lieu seulement sur le plan des identités religieuses mais également au niveau scientifique. Un fragment conservé par Héphaistion pose une alternative entre les recommandations d'Hermès Trismégiste et la méthode proposée par l'astrologue Dorothée ${ }^{133}$. Précisons qu'il ne s'agit pas de la première attestation païenne de l'épithète, qui apparaît en fait dans le contexte de la citation et non dans la citation elle-même, ce qui la place donc au IV siècle. En suivant l'ordre chronologique,

internazionale per lo studio dei papiri ercolanesi, 1984, p. 683-693 ; Emilio Suárez de la Torre, « Mito, teología, magia y astrología en PGM XIII (P. Leid. J 395) », in Mito y magia en Grecia y Roma, éds. Emilio Suarez de la Torre et Aurelio Pérez Jiménez, Zaragoza, Espagne, Libros Pórtico, 2013, p. 179-202 ; pour le rattachement à l'Égypte, voir Reinhold Merkelbach, Abrasax: ausgewählte Papyri religiösen und magischen Inhalts. Band 3, Zwei griechisch-ägyptische Weihezeremonien : die Leidener Weltschöpfung, die Pschai-Aion-Liturgie, Opladen, Allemagne, Westdeutscher, 1992 ; pour les rapports au judaïsme, Bert Jan Lietaert Peerbolte, «The Eighth Book of Moses (PLeid. J 395) : Hellenistic Jewish Influence in a Pagan Magical Papyrus », A Kind of Magic. Understanding Magic in the New Testament and its Religious Environment, éds. Michael Labahn et Bert Jan Lietaert Peerbolte, 2007, p. 184-194 ; Todd E. Klutz, « The Eighth Book of Moses. A new translation and introduction », in Old Testament pseudepigrapha : more noncanonical scriptures, I, éds. Richard Bauckham, James R Davila et Alexander Panayotov, 2013, p. 189-235.

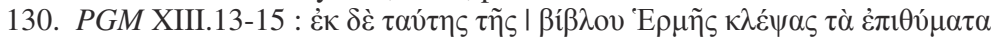

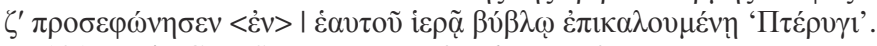

131. Voir Guy Stroumsa, La fin du sacrifice. Les mutations religieuses de l'Antiquité tardive, Paris, Odile Jacob, 2005, p. 63-101.

132. Sur ce point, voir Kerchove, loc. cit.

133. Dorothée, fr. 2e3 (éd. Pingree Carmen Astrologicum). 
les titres de l'écrit attribué au médecin Thessalus font mention du Trismégiste ${ }^{134}$ mais la nature du texte n'autorise pas à établir avec certitude une datation au $\mathrm{I}^{\mathrm{er}}$ siècle. En revanche, le trajet de la narration (de la Grèce vers Thèbes) rend perceptible la volonté de défendre la primauté de l'Égypte dans l'ordre du savoir ${ }^{135}$. Les premières attestations païennes se trouveraient donc peutêtre chez l'astrologue Thrassylus, chez Philon de Byblos et dans les Cyranides ${ }^{136}$, vers les $\mathrm{I}^{\text {er }}$ et $\mathrm{II}^{\mathrm{e}}$ siècles. La première citation d'Hermès Trismégiste en latin, chez Tertullien, fait justement d'Hermès le «maître de tous les physiciens » (magister omnium physicorum $)^{137}$. On sait que «Chaldéens » et « Égyptiens » incarnaient deux branches de l'astrologie hellénistique parfois en désaccord ${ }^{138}$. Le nom d'Hermès en astrologie pouvait ainsi être une stratégie rhétorique pour défendre la supériorité de l'Égypte sur sa rivale, comme c'était le cas avec les noms de Pétosiris et Néchepso.

Alors que les auteurs païens faisaient de la tradition un gage d'autorité et citent donc plus volontiers «les Égyptiens» (oi Aîvó $\pi \tau 101$ ), la révélation devient le critère essentiel pour les nouveaux courants religieux et le prophète le garant ultime de l'autorité. L'égyptien Chérémon pouvait passer pour stoïcien aux yeux des Romains et peut-être était-ce avec lui la philosophie égyptienne qui passait également pour telle ${ }^{139}$ mais désormais une équivalence philosophique ne suffit pas et une figure personnelle apparaît nécessaire pour porter une révélation en même temps

134. Thessalus, De virtutibus herbarum I prol. ; I, 25 (éd. Friedrich).

135. Sur Thessalus, voir André-Jean Festugière, «L'expérience religieuse du médecin Thessalos », Revue biblique, 1939, p. 45-77 ; Ioan Moyer, «Thessalos of Tralles and cultural exchange ", in Prayer, magic, and the stars in the ancient and late antique world, éds. Joel Thomas Walker, Brannon Wheeler et Scott B. Noegel, Pennsylvania State University Pr., 2003, p. 39-56.

136. Catalogus Codicum Astrologorum Graecorum 8,3 p. 101.17 ; Cyranides I prol., 7 ; I, 4.52 ; Eusèbe, Préparation Évangélique, I, 10.17.

137. Tertullien, Contre les Valentiniens, 15 (Kroymann p. 194).

138. Voir par exemple Ptolémée, Apotélesmatique, I, 21, sur les théories égyptienne et chaldéenne des confins. Pour établir le bien-fondé de sa propre théorie, Ptolémée prétend la tenir «d'un manuscrit ancien en grande partie

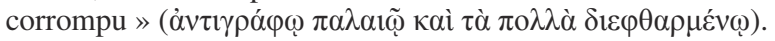

139. Porphyre, De l'abstinence, IV, 6.2 ; Contra christianos, fr. 39.33 (éd. Harnak). Voir John Gee, « Review of Hans Dieter Betz, The "Mithras Liturgy" : Text, Translation and Commentary », [En ligne : https://www.bookreviews.org/ pdf/4294_4269.pdf]. Consulté le 22 octobre 2017. 
qu'une identité. Il est possible que l'élaboration d'Hermès Trismégiste en figure prophétique soit une réponse gréco-égyptienne à un problème posé en des termes nouveaux : à partir du moment où la vérité fait l'objet de la religion, où le livre tient lieu de médiation et où une figure personnelle incarne l'autorité, il devient nécessaire de reformuler son identité au sein du paradigme qui s'impose progressivement sur le bassin méditerranéen. Deux phénomènes sont donc à distinguer : d'un côté la diffusion, à partir de l'époque hellénistique, d'une littérature scientifique et philosophique (astrologie, alchimie, magie...) proposant de nouvelles conceptions de la nature et de l'univers; de l'autre, la construction d'une autorité religieuse dans une logique de compétition identitaire au sein d'une société hellénistique multiculturelle. D'un côté, la circulation des savoirs a conduit à leur partage interculturel et à leur complexification. De l'autre, les problématiques de positionnement identitaire ont conduit à des appropriations et à la mise en place d'une compétition pour la vérité. Le nom d'Hermès Trismégiste vient alors recouvrir un phénomène contemporain pour le convertir en moyen d'action dans la promotion d'une identité. Il permet à la fois la synthèse (des savoirs) et la concurrence (des identités). La circulation du nom divin met ainsi en lumière des effets de pouvoir. Il servait à l'exaltation d'une puissance divine locale et à la mise en acte d'une efficacité rituelle. Mais ces deux aspects sont reconvertis pour constituer une identité collective derrière une figure d'autorité sur le plan religieux et scientifique. Le nom divin donne alors du pouvoir aux communautés qui s'en revendiquent pour des processus de différenciation ${ }^{140 *}$.

florian.audureau@orange.fr

140. Lors de la préparation de cet article, je n'ai pas pu consulter la publication récente de Christian $\mathrm{H}$. Bull qui était encore sous presse et que je recommande maintenant : The tradition of Hermes Trismegistus : the Egyptian priestly figure as a teacher of Hellenized wisdom, Leyde-Boston, Brill (« Religions in the GraecoRoman world»), 2018.

* Je remercie Mmes Anna Van den Kerchove et Nicole Belayche pour leur relecture d'une première version de ce travail, M. Jean-Michel Roessli pour ses encouragements et la Revue pour ses précieuses remarques. Il va de soi que je prends l'entière responsabilité des arguments développés dans cet article sous sa nouvelle version. 